\title{
JOSEF NEDOMA
}

\section{The finite element solution of elliptic and parabolic equations using simplicial isoparametric elements}

RAIRO - Analyse numérique, tome 13, no 3 (1979), p. 257-289.

<http://www.numdam.org/item?id=M2AN_1979_13_3_257_0>

(C) AFCET, 1979, tous droits réservés.

L'accès aux archives de la revue «RAIRO - Analyse numérique » implique l'accord avec les conditions générales d'utilisation (http://www.numdam.org/ legal.php). Toute utilisation commerciale ou impression systématique est constitutive d'une infraction pénale. Toute copie ou impression de ce fichier doit contenir la présente mention de copyright.

\section{Numdam}




\title{
THE FINITE ELEMENT SOLUTION OF ELLIPTIC AND PARABOLIC EQUATIONS USING SIMPLICIAL ISOPARAMETRIC ELEMENTS ( ${ }^{*}$ )
}

\author{
par Josef Nedoma $\left({ }^{1}\right)$ \\ Communiqué par P. G. Ciarlet
}

\begin{abstract}
Error bounds introduced in [7] were given for fully discretized approximate solutions of parabolic equations by the finite element method. For time discretization the A-stable linear v-step methods ( for $v=1$ or 2) were used. In this paper the $A_{0}$-stable linear $v$-step methods for any $v$ are used for time discretization. It is known that $A$-stable methods for $v=1,2$ are included in the class of $\boldsymbol{A}_{0}$-stable methods. The consideration for the elliptic equations is similar to the parabolic equations. Hence, the error bounds for elliptic equations are formulated in this paper too.
\end{abstract}

Résumé. - On a donné en [7] des majorations de l'erreur pour des approximations complètement discrètes d'équations paraboliques par la méthode des éléments finis. On utilisait des méthodes linéaires $A$-stables à $v$ pas $(v=1$ ou 2$)$ pour la discrétisation en temps. Dans cet article, on utilise des méthodes linéaires $A_{0}$-stables à $\vee$ pas, $\vee$ quelconque, pour la discrétisation en temps. On sait que les méthodes $A$-stables pour $v=1,2$ sont incluses dans la classe des méthodes $A_{0}$-stables. Les développements étant semblables dans les cas elliptiques et paraboliques, on énonce également dans cet article les majorations d'erreurs pour les équations elliptiques.

\section{CONSTRUCTION OF THE FINITE ELEMENT SPACE. NOTATION}

We consider the $k$-regular family $\{K\}_{h}$ of simplicial isoparametric finite elements $K$ introduced by Ciarlet and Raviart [3]. Hence, the simplicial element $K \in\{K\}_{h}$ is the image of the unit $n$-simplex $\hat{K}(\hat{K}$ is the closed convex hull of a set $\left.\hat{\Sigma}=\bigcup_{i=1}^{N}\left\{\hat{a}_{i}\right\}\right)$ through the unique mapping $F_{K}: \hat{K} \rightarrow R^{n}$ (the mapping $F_{K}$ is supposed to be a $C^{k+1}$-diffeomorphism) such that $F_{K} \in \hat{P}^{n}, \quad F_{K}\left(\hat{a}_{i}\right)=a_{i}$ $\left(\hat{P} \subset C^{k+1}(\hat{K})\right.$ is a finite dimensional space of functions defined on $\hat{K}$ with

(*) Reçu septembre 1978.

(1) Technical University, Laboratoř počítacích strojů V.U.T., Brno, Tchécoslovaquie.

R.A.I.R.O. Analyse numérique/Numerical Analysis, 0399-0516/1979/257/\$4.00

(C) Bordas-Dunod 
$\operatorname{dim} \hat{P}=N$ such that $\hat{\Sigma}$ is $\hat{P}$-unisolvent and $\hat{P} \supset \hat{P}(1)$, where for any integer $r \geqq 0, \hat{P}(r)$ is the space of restrictions to $\hat{K}$ of all polynomials of degree $\leqq r$ in $n$ variables $\hat{x}_{1}, \ldots, \hat{x}_{n}$ ) and there exist constants $c_{i}, 0 \leqq i \leqq k+1$, independent of $h$ such that for all $h$ :

$$
\sup _{\hat{x} \in \mathbb{R}} \max _{|\alpha|=l}\left|D^{\alpha} F_{K}(\hat{x})\right| \leqq c_{i} h^{2}, \quad 1 \leqq i \leqq k+1
$$

and

$$
0<\frac{1}{c_{0}} h^{n} \leqq\left|J_{K}(\hat{x})\right| \leqq c_{0} h^{n},
$$

where $\alpha=\left(\alpha_{1}, \ldots, \alpha_{n}\right),|\alpha|=\alpha_{1}+\ldots+\alpha_{n}$ and $J_{K}(\hat{x})$ is the Jacobian of the mapping $F_{K}$ at the point $\hat{x} \in \hat{K}$.

To every element $K$ there is associated the finite dimensional space $P_{K}$ (with $\operatorname{dim} P_{K}=N$ ) of functions

$$
P_{K}=\left\{p_{K}: K \rightarrow R ; p_{K}=p^{*}\left(F_{K}^{-1}\right), \forall p^{*} \in \hat{P}\right\} .
$$

The $K$-interpolate $\pi_{K} u$ of a given function $u: K \rightarrow R$ is the unique function which satisfies

$$
\pi_{K} u \in P_{K}, \quad \pi_{K} u\left(a_{i}\right)=u\left(a_{i}\right), \quad 1 \leqq i \leqq N .
$$

For a $k$-regular family $\{K\}_{h}$ of finite elements the following interpolation theorem is true (see Ciarlet and Raviart [3], theorem 2, p. 429).

LEMMA 1.1 (interpolation theorem): Let a $k$-regular family $\{K\}_{h}$ of simplicial elements such that $\hat{P}(k) \subset \hat{P}$ be given. Let

$$
k>\frac{n}{2}-1 \text {. }
$$

Then for any integer $i$ such that $0 \leqq i \leqq k+1$, there exists a constant $c$ independent of $h$ such that for any $K \in\{K\}_{h}$ and for any function $u \in H^{k+1}(K)$ we have

$$
\left|u-\pi_{K} u\right|_{\imath, K} \leqq c h^{k+1-i}\|u\|_{k+1, K} .
$$

Here the following notation is used:

The norm and the scalar product in the space $L^{2}(A)$ is denoted by $\|\cdot\|_{0, A}$ and $(.,)_{0, A}$ respectively.

$H^{m}(A) \equiv W_{2}^{(m)}(A), m=0,1 \ldots$ is a Sobolev space with the norm

$$
\|v\|_{m, A}=\left(\sum_{i=0}^{m}|v|_{i, A}^{2}\right)^{1 / 2}, \quad \text { where } \quad|v|_{i, A}=\left(\sum_{|\alpha|=t}\left\|D^{\alpha} v\right\|_{0, A}^{2}\right)^{1 / 2}
$$

R.A.I.R.O. Analyse numérique/Numerical Analysis 
In the sequel we mean by $\Omega$ a bounded domain in $R^{n}$ with a sufficiently smooth boundary $\partial \Omega$.

Using the way described by Ciarlet and Raviart [3] we define a $k$-regular triangulation $\mathscr{C}_{h}$ of $\Omega$. Let $\Omega_{h}$ be the union of a finite number of simplicial elements $K$. Every element $K=F_{K}(\hat{K})$ is determined by $N$ points $a_{\imath, K}$. We suppose that all points $a_{i, K}$ belong to $\bar{\Omega}$. The family of elements constructed in this way is called a triangulation of $\Omega$ and is denoted by $\mathscr{C}_{h}$. We say that a triangulation $\mathscr{C}_{h}$ of $\Omega$ is $k$-regular if:

a) the family of all elements from which the triangulation is formed is $k$-regular;

b) the geometrical shape of any "face" $\Delta$ of a given element $K \in \mathscr{C}_{h}$ must be completely determined by those points $a_{i, K}$ which belong to $\Delta$;

c) for the boundary elements (i.e. for elements $K \nsubseteq \bar{\Omega}$ ) of the triangulation $\mathscr{C}_{h}$ we have

$$
\max _{y^{\prime} \in \Delta}\left|\psi_{h}\left(y^{\prime}\right)-\psi\left(y^{\prime}\right)\right| \leqq c h^{k+1}
$$

where $c$ is a constant independent of $h$ and the notation is that of figure.

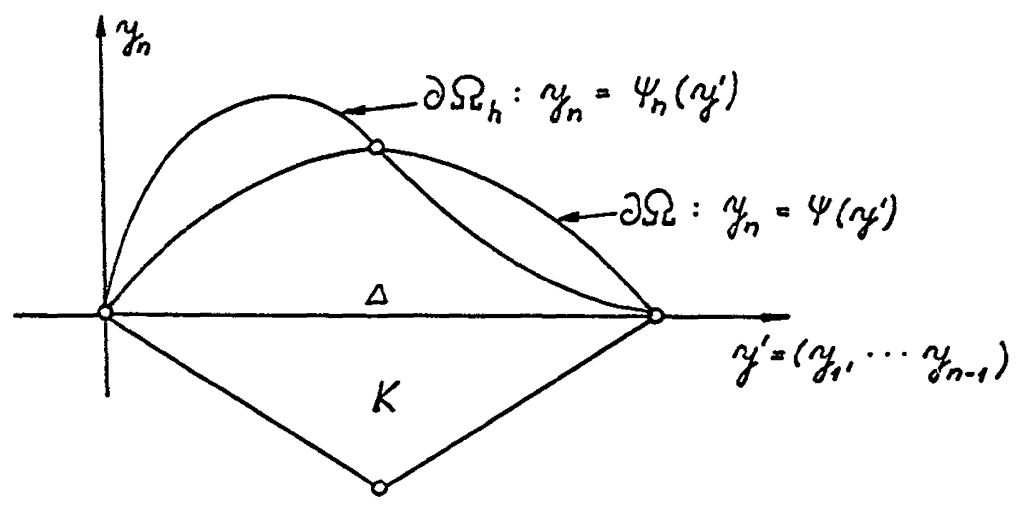

To a given $k$-regular triangulation $\mathscr{C}_{h}$ there is associated the finite dimensional space $V_{h}$ of functions $v$ defined by

$$
V_{h}=\left\{v \in C^{0}\left(\bar{\Omega}_{h}\right) ; v_{K} \in P_{K}, \forall K \in \mathscr{C}_{h}, v=0 \text { on } \partial \Omega_{h}\right\},
$$

where $v_{K}$ is the restriction of the function $v$ to the set $K$.

vol. $13, \mathrm{n}^{\circ} 3,1979$ 
Next, to any function $v$ defined on $\bar{\Omega}$ or on $\bar{\Omega}_{h}$ we may associate its unique interpolate $\pi_{h} v$, which satisfies

$$
\pi_{h} v=\pi_{K} v, \quad \forall K \in \mathscr{C}_{h} .
$$

In our paper we suppose that $\hat{P} \equiv \hat{P}(k)$. This restriction is not essential. It enables us to give simpler proofs.

In the sequel we use the following notation:

$H_{0}^{1}(A)$ is the closure of the set $C_{0}^{\infty}(A)$ (i. e. of the set of infinitely differentiable functions with compact support in $A$ ) in the norm $\|\cdot\|_{1, A}$.

$H^{-1}(A)$ is the space dual to $H_{0}^{1}(A)$ (with dual norm).

$L^{\infty}\left(H^{m}(A)\right)$ is the space of all functions $\varphi(x, t), x=\left(x_{1}, \ldots, x_{n}\right) \in A, t \in[0, T]$ such that $\varphi(x, t) \in H^{m}(A), \forall t \in[0, T]$ and the function $\|\varphi(x, t)\|_{m, A}$ is bounded for almost all $t \in[0, T]$.

Let $\Phi(x)$ be any function defined on the element $K$. Then the function $\Phi\left(F_{K}(\hat{x})\right)$ is defined on $\hat{K}$. In the sequel we will denote it by $\Phi^{*}(\hat{x})$.

In the sequel the constants independent of $h$ will be denoted by $c$. The notation is generic, i. e. $c$ will not denote necessarily the same constant in any two places.

\section{ISOPARAMETRIC INTEGRATION}

In the same way as in Ciarlet and Raviart [3] let us suppose that we have at our disposal a quadrature formula of degree $d$ over the reference set $\hat{K}$. In other words

$$
\int_{R} \varphi(\hat{x}) d \hat{x} \text { is approximated by } \sum_{r} \hat{\omega}_{r} \varphi\left(\hat{b}_{r}\right)
$$

for some specified points $\hat{b}_{r} \in \hat{K}$ and weights $\hat{\omega}_{r}$ which will be assumed once and for all to satisfy

$$
\hat{\omega}_{r}>0 \text {. }
$$

This assumption is by no means necessary but it yields simpler proofs. Concerning $\hat{b}_{r}$ we suppose that for every $r, \hat{b}_{r}$ either lies inside $\hat{K}$ or it coincides with some of the points $\hat{a}_{i}$. With the quadrature scheme (2.1) we associate the error

$$
\hat{E}(\varphi)=\int_{K} \varphi(\hat{x}) d \hat{x}-\sum_{r} \hat{\omega}_{r} \varphi\left(\hat{b}_{r}\right)
$$


Using the standard formula for change of variables in multiple integrals, we find that

$$
\int_{K} \varphi(x) d x \text { is approximated by } \sum_{r} \omega_{r, \mathrm{~K}} \varphi\left(b_{r, \mathrm{~K}}\right)
$$

where

$$
\omega_{r, K}=\hat{\omega}_{r} J_{K}\left(\hat{b}_{r}\right), \quad b_{r, K}=F_{K}\left(\hat{b}_{r}\right) .
$$

We may, and will, assume that $J_{K}(\hat{x})>0, \forall \hat{x} \in \hat{K}$. We see that the quadrature scheme (2.1) over the reference set $\hat{K}$ induces the quadrature scheme (2.4) over the element $K$, a circumstance which is called by Ciarlet and Raviart [3] "isoparametric numerical integration". With the scheme (2.4) we associate the error

$$
E_{K}(\varphi)=\int_{K} \varphi(x) d x-\sum_{r} \omega_{r, K} \varphi\left(b_{r, K}\right)
$$

so that we have

$$
E_{K}(\varphi)=\hat{E}\left(\varphi^{*} J_{K}\right) \quad \text { and } \quad \hat{E}\left(\varphi^{*}\right)=E_{K}\left(\varphi J_{K}^{-1}\right) .
$$

In the sequel we will denote

$$
E(\varphi)=\sum_{K \in \mathscr{Q}_{n}} E_{K}(\varphi) \text { for any function } \varphi
$$

Now, we derive two theorems concerning isoparametric numerical integration. Before, we give some technical lemmas.

Lemma 2.1: Let $D^{\beta} \varphi_{i}=O\left(h^{|\beta|+\mathscr{H}_{i}}\right)$ for $i=1, \ldots, s,|\beta|=0, \ldots,|\alpha|$. Then

$$
D^{\alpha}\left(\varphi_{1} \varphi_{2} \ldots \varphi_{s}\right)=O\left(h^{|\alpha|+\mathscr{H}_{1}+\ldots+\mathscr{H}_{s}}\right) .
$$

The proof is trivial using the mathematical induction.

Lemma 2.2: For polynomials $r, s$ on the reference set $\hat{K}$ the following inequalities are true

$$
\begin{gathered}
\max _{\hat{K}}\left|D^{\alpha} r\right| \leqq c_{1}|r|_{|\alpha|, \mathbb{R}}, \\
|r|_{j, \mathbb{R}}^{2} \leqq c_{2}|r|_{i, \mathbb{R}}^{2} \quad \text { for } \quad j \geqq i \geqq 0, \\
|r s|_{i, \mathbb{K} \leqq}^{2} \leqq c_{3} \sum_{j=0}^{i}|r|_{j, \mathbb{K}}^{2}|s|_{i-j, \mathbb{K}}^{2},
\end{gathered}
$$

where $c_{1}, c_{2}, c_{3}$ are constants.

vol. $13, \mathrm{n}^{\circ} 3,1979$ 
The proof follows from Zlámal's paper [12], p. 356 and from lemma 3 in [7].

LeMMA 2.3: Let $\mathscr{C}_{h}$ be a k-regular triangulation of $\Omega$. Let $J_{K}^{(r, p)}$ be a cofactor of the Jacobian $J_{K}$. Then

$$
\begin{gathered}
D^{\alpha} J_{K}=O\left(h^{|\alpha|+n}\right), \\
D^{\alpha} J_{K}^{(r, p)}=O\left(h^{|\alpha|+n-1}\right), \\
D^{\alpha}\left(\frac{1}{J_{K}}\right)=O\left(h^{|\alpha|-n}\right) .
\end{gathered}
$$

For the proof see Lemma 5 in [7].

Lemma 2.4: Let $\tau^{*} \in H^{k+1}(\hat{K}), \tau \in H^{k+1}(K), K \in \mathscr{C}_{h}, \mathscr{C}_{h}$ be a $k$-regular triangulation of $\Omega$. Then there exists a constant $c$ independent of $h$ such that

$$
\left|\tau^{*}\right|_{k+1, K} \leqq c h^{-(n / 2)+k+1}\|\tau\|_{k+1, K} .
$$

Lemma is an immediate consequence of Lemma 1 from [3], p. 427.

Lemma 2.5: Let $\varphi \in H^{s}(\hat{K})$, where $s>n / 2$ and let $\pi_{s-1} \varphi$ be a polynomial of degree $s-1$ which uniquely interpolates the function $\varphi$ on $\hat{K}$. Then there exists a constant $c$ such that

$$
\left|\varphi-\pi_{s-1} \varphi\right|_{, R} \leqq c|\varphi|_{s, \mathbb{R}} \quad \text { for } \quad j=0, \ldots, s .
$$

Lemma follows from Bramble and Hilbert paper [2], p. 812.

LEMMA 2.6: Let $\psi(\hat{x}) \in H^{s}(\hat{K})$, where

$$
s>\frac{n}{2},
$$

$\tau(\hat{x})$ be a polynomial of degree $\leqq r$, where

$$
r \leqq s
$$

$\delta(\hat{x}) \in C^{s}(\hat{K})$ be a function such that

$$
D^{\alpha} \delta=O\left(h^{|\alpha|+\mathscr{H}}\right) \quad \text { for } 0 \leqq|\alpha| \leqq s, \quad \mathscr{H} \ldots \text { some int. }
$$

Let $d$ be the order of a quadrature formula on the reference set $\hat{K}$ such that

$$
d>\frac{n}{2}-1
$$

R.A.I.R.O. Analyse numérique/Numerical Analysis 
Then there exists a constant $c$ such that

$$
\begin{aligned}
|\hat{E}(\delta \psi \tau)|^{2} & \leqq c h^{2 \mathscr{E}}\left\{\left(h^{2 s}\|\psi\|_{s, \mathrm{~K}}^{2}+|\psi|_{s, \mathbb{R}}^{2}\right)\|\tau\|_{0, \mathbb{R}}^{2}\right. \\
& \left.+h^{2(d+1)}\left(\sum_{i=0}^{r} h^{-2 i}|\tau|_{i, K}^{2}\right)\left(h^{-2(s-1)}|\psi|_{s, \mathbb{R}}^{2}+\sum_{i=0}^{s-1} h^{-2 i}|\psi|_{2, \mathbb{R}}^{2}\right)\right\} .
\end{aligned}
$$

When supposing, in addition, that $\psi(\hat{x})$ is a polynomial of degree $\leqq r$, then there exists a constant $c$ such that

$$
\begin{aligned}
\left|\hat{E}\left(\delta \frac{\partial \psi}{\partial \hat{x}_{i}} \frac{\partial \tau}{\partial \hat{x}_{j}}\right)\right|^{2} \leqq c h^{2 \mathscr{H}}\left\{h^{2 s} \|\right. & \psi \|_{0, \mathrm{R}}^{2}|\tau|_{1, K}^{2} \\
& \left.+h^{2(d+3)} \sum_{i=1}^{r} h^{-2 i}|\psi|_{i, \mathbb{R}}^{2} \sum_{i=1}^{r} h^{-2 i}|\tau|_{i, \mathbb{R}}^{2}\right\} .
\end{aligned}
$$

Proof: Evidently

$$
\begin{array}{r}
|\hat{E}(\delta \psi \tau)| \leqq\left|\hat{E}\left(\left(\delta-\pi_{s-1} \delta\right)\left(\psi-\pi_{s-1} \psi\right) \tau\right)\right|+\left|\hat{E}\left(\left(\delta-\pi_{s-1} \delta\right) \pi_{s-1} \psi \tau\right)\right| \\
+\left|\hat{E}\left(\pi_{s-1} \delta\left(\psi-\pi_{s-1} \psi\right) \tau\right)\right|+\left|\hat{E}\left(\pi_{s-1} \delta \pi_{s-1} \psi \tau\right)\right| .
\end{array}
$$

From (2.3), from the first Sobolev theorem and from lemma 2.5 it follows

$$
\begin{aligned}
\mid \hat{E}\left(\left(\delta-\pi_{s-1} \delta\right)(\psi\right. & \left.\left.-\pi_{s-1} \psi\right) \tau\right) \mid \\
& \leqq c \sup _{\mathcal{K}}\left|\delta-\pi_{s-1} \delta\right| \sup _{R}\left|\psi-\pi_{s-1} \psi\right| \max _{R}|\tau| \\
& \leqq c\left\|\delta-\pi_{s-1} \delta\right\|_{s, R}\left\|\psi-\pi_{s-1} \psi\right\|_{s, \mathbb{R}} \max _{R}|\tau| \\
& \leqq c|\delta|_{s, R}|\psi|_{s, \hat{K}} \max _{K}|\tau| .
\end{aligned}
$$

Hence, from (2.20) and from lemma 2.2 we get

$$
\left|\hat{E}\left(\left(\delta-\pi_{s-1} \delta\right)\left(\psi-\pi_{s-1} \psi\right) \tau\right)\right|^{2} \leqq c h^{2(s+\mathscr{C})}|\psi|_{s, K}^{2}\|\tau\|_{0, K}^{2}
$$

Similarly we obtain

$$
\begin{gathered}
\left|\hat{E}\left(\left(\delta-\pi_{s-1} \delta\right) \pi_{s-1} \psi \tau\right)\right|^{2} \leqq c h^{2(s+\mathscr{H})}\|\psi\|_{s, \mathbb{R}}^{2}\|\tau\|_{0, \mathbb{R}}^{2} \\
\left|\hat{E}\left(\pi_{s-1} \delta\left(\psi-\pi_{s-1} \psi\right) \tau\right)\right|^{i} \leqq c h^{2 \mathscr{H}}|\psi|_{s, \mathbb{R}}^{2}\|\tau\|_{0, R}^{2} .
\end{gathered}
$$

Evidently

$$
\left|\pi_{s-1} \varphi\right|_{i, \mathbb{K}} \leqq\left|\pi_{s-1} \varphi-\varphi\right|_{i, \mathbb{K}}+|\varphi|_{i, \mathbb{K}} \leqq c\left(|\varphi|_{s, \mathbb{K}}+|\varphi|_{i, \mathbb{R}}\right), \quad 0 \leqq i \leqq s .
$$




\section{Hence}

$$
\begin{gathered}
\left|\pi_{s-1} \psi\right|_{i, \mathbb{R}} \leqq c\left(|\psi|_{s, \mathbb{R}}+|\psi|_{i, \mathbb{R}}\right), \quad 0 \leqq i \leqq s, \\
\left|\pi_{s-1} \delta\right|_{i, \mathbb{R}} \leqq c\left(|\delta|_{s, \mathbb{R}}+|\delta|_{i, \mathbb{R}} \leqq c\left(h^{s+\mathscr{H}}+h^{i+\mathscr{H}}\right) \leqq c h^{i+\mathscr{H}} .\right.
\end{gathered}
$$

Let us remember that the inequality (2.29) is true also for $i>s$ since $\left|\pi_{s-1} \delta\right|_{, . \tilde{K}}=0$. From the Bramble-Hilbert lemma (see [1]), from lemma 2.2 and from (2.29) we get

$$
\begin{aligned}
\left|\hat{E}\left(\pi_{s-1} \delta \pi_{s-1} \psi \tau\right)\right|^{2} \leqq & c\left|\pi_{s-1} \delta \pi_{s-1} \psi \tau\right|_{d+1, R}^{2} \\
& \leqq c \sum_{j=0}^{d+1}\left|\tau \pi_{s-1} \psi\right|_{j, K}^{2}\left|\pi_{s-1} \delta\right|_{d+1-j, R}^{2} \\
& \leqq c h^{2(\mathscr{H}+d+1)} \sum_{j=0}^{2 s} h^{-2 j}\left|\tau \pi_{s-1} \psi\right|_{j, R}^{2} \\
& \leqq c h^{2(\mathscr{H}+d+1)} \sum_{j=0}^{2 s} h^{-2 j} \sum_{i=0}^{j}|\tau|_{i, \mathbb{R}}^{2}\left|\pi_{s-1} \psi\right|_{j-1, \hat{K}}^{2} .
\end{aligned}
$$

It is easy to verify that

$$
\sum_{j=0}^{2 s} h^{-2 j} \sum_{i=0}^{j}|\tau|_{i, K}^{2}\left|\pi_{s-1} \psi\right|_{j-1, \mathbb{K}}^{2}=\left(\sum_{j=0}^{s} h^{-2 j}|\tau|_{j, K}^{2}\right)\left(\sum_{j=0}^{s} h^{-2 j}\left|\pi_{s-1} \psi\right|_{j, \hat{K}}^{2}\right) .
$$

Hence, from (2.30) and from (2.28) it follows

$$
\begin{aligned}
& \mid \hat{E}\left(\left.\pi_{s-1} \delta \pi_{s-1} \psi \tau\right|^{2}\right. \\
& \quad \leqq c h^{2(\mathscr{H}+d+1)}\left(\sum_{j=0}^{r} h^{-2 j}|\tau|_{j, \mathrm{~K}}^{2}\right)\left(h^{-2(s-1)}|\psi|_{s, R}^{2}+\sum_{j=0}^{s-1} h^{-2 j}|\psi|_{j, K}^{2}\right)
\end{aligned}
$$

Substituting from (2.25), (2.26), (2.27) and from (2.31) into (2.24) we get (2.22). From (2.22), it follows

$$
\begin{aligned}
& \left|\hat{E}\left(\delta \frac{\partial \psi}{\partial \hat{x}_{i}} \frac{\partial \tau}{\partial \hat{x}_{j}}\right)\right| \leqq c h^{2 \mathscr{H}}\left\{\left(h^{2 s}\|\psi\|_{s+1, R}^{2}+|\psi|_{s+1, R}^{2}\right)|\tau|_{1, R}^{2}\right. \\
& \left.+h^{2(d+1)}\left(\sum_{i=0}^{s} h^{-2 i}|\tau|_{i+1, R}^{2}\right)\left(h^{-2(s-1)}|\psi|_{s+1, R}^{2}+\sum_{i=0}^{s-1} h^{-2 i}|\psi|_{i+1, R}^{2}\right)\right\} .
\end{aligned}
$$

If $\psi(\hat{x})$ is a polynomial of degree $\leqq r(r \leqq s)$ then

$$
\|\psi\|_{s+1, R}^{2} \leqq c\|\psi\|_{0, R}^{2} \quad \text { and } \quad|\psi|_{s+1, R}^{2}=0 .
$$


Evidently

$$
\begin{aligned}
& \sum_{i=0}^{r} h^{-2 i}|\tau|_{i+1, \mathbb{R}}^{2}=\sum_{i=1}^{r+1} h^{-2(i-1)}|\tau|_{i, \mathbb{R}}^{2}=h^{2} \sum_{i=1}^{r} h^{-2 i}|\tau|_{i, \mathbb{R}}^{2}, \\
& \sum_{i=0}^{s-1} h^{-2 i}|\psi|_{i+1, R}^{2}=\sum_{i=1}^{s} h^{-2(i-1)}|\psi|_{i, \mathbb{R}}^{2}=h^{2} \sum_{i=1}^{r} h^{-2 i}|\psi|_{i, \mathrm{R}}^{2} .
\end{aligned}
$$

Substituting from (2.33)-(2.35) into (2.32) we get (2.23).

Now we can formulate two theorems concerning isoparametric integration.

THeOREM 2.1: Let $\mathscr{C}_{h}$ be a $k$-regular triangulation of the set $\Omega$ where

$$
k>\frac{n}{2}-1 \text {. }
$$

Let $v \in V_{h}\left(\Omega_{h}\right)$ and $\varphi \in H^{m}\left(\Omega_{h}\right)$, where

$$
m=\max \left(\left[\frac{n}{2}\right]+1, k\right) .
$$

Let the quadrature formula given on the reference set $\hat{K}$ be of degree

$$
d \geqq \max (1,2 k-2) \text {. }
$$

Then there exists a constant $c$ such that

$$
|E(\varphi v)| \leqq c h^{k}\|\varphi\|_{m, \Omega_{*}}\|v\|_{1, \Omega_{*}} .
$$

If, in addition, $\varphi \in H^{k+1}\left(\Omega_{h}\right)$ then there exists a constant $c$ such that

$$
|E(\varphi v)| \leqq c h^{k+1}\|\varphi\|_{k+1, \Omega_{k}}\left(\sum_{K \in \mathcal{Q}_{1}}\|v\|_{2, K}^{2}\right)^{1 / 2} .
$$

Proof: Obviously

$$
E(\varphi v)=\sum_{K \in \mathcal{Q}_{\Lambda}} E_{K}(\varphi v)=\sum_{K \in \mathcal{Q}_{n}} \hat{E}\left(J_{K} \varphi^{*} v^{*}\right)
$$

It is easy to verify that $m>n / 2, k \leqq m \leqq k+1, \max (1,2 k-2) \geqq k$ and that $D^{\alpha}\left(J_{K}\right)=O\left(h^{|\alpha|+n}\right)$. Hence, we may apply Lemma 2.6 for $\psi=\varphi^{*}, s=m, \tau=v^{*}$, $r=k, \delta=J_{K}, \mathscr{H}=n$ and $d \geqq \max (1,2 k-2)$. From (2.22) we get

$$
\begin{aligned}
&\left|\hat{E}\left(J_{K} \varphi^{*} v^{*}\right)\right|^{2} \leqq c h^{2 n}\left\{\left(h^{2 m}\left\|\varphi^{*}\right\|_{m, \mathrm{~K}}^{2}+\left|\varphi^{*}\right|_{m, \mathrm{~K}}^{2}\right)\left\|v^{*}\right\|_{0, \mathrm{~K}}^{2}\right. \\
&\left.+h^{2(d+1)}\left(\sum_{i=0}^{k} h^{-2 i}\left|v^{*}\right|_{i, \mathrm{~K}}^{2}\right)\left(h^{-2(m-1)}\left|\varphi^{*}\right|_{m, \mathrm{R}}^{2}+\sum_{i=0}^{m-1} h^{-2 i}\left|\varphi^{*}\right|_{i, \mathrm{R}}^{2}\right)\right\} .
\end{aligned}
$$


Hence from Lemma 2.4 (notice that a $k$-regular family is a $k^{\prime}$-regular family for any $k^{\prime} \leqq k$ ) it follows

$$
\begin{aligned}
\left|\hat{E}\left(J_{K} \varphi^{*} v^{*}\right)\right|^{2} \leqq c\left\{h^{2 m}\|v\|_{0, K}^{2} \|\right. & \varphi \|_{m, K}^{2} \\
& \left.+h^{2(d+1)}\|\varphi\|_{m, K}^{2} h^{n} \sum_{i=0}^{k} h^{-2 i}\left|v^{*}\right|_{i, K}^{2}\right\} .
\end{aligned}
$$

In the same manner we may apply the inequality (2.22) for $s=k+1$ assuming $\varphi \in H^{k+1}\left(\Omega_{h}\right)$. Then we obtain

$$
\begin{aligned}
\left|\hat{E}\left(J_{K} \varphi^{*} v^{*}\right)\right|^{2} \leqq c\left\{h^{2(k+1)}\|v\|_{0, K}^{2}\|\varphi\|_{k+1, K}^{2}\right. & \\
& \left.+h^{2(d+1)}\|\varphi\|_{k+1, K}^{2} h^{n} \sum_{i=0}^{k} h^{-2 i}\left|v^{*}\right|_{i, K}^{2}\right\} .
\end{aligned}
$$

From lemma 2.2 and from lemma 2.4 we get for $k \geqq 1$ :

$$
\begin{aligned}
\sum_{i=0}^{k} h^{-2 i}\left|v^{*}\right|_{i, \mathrm{R}}^{2}=\left|v^{*}\right|_{0, \mathrm{R}}^{2}+\sum_{i=1}^{k} h^{-2 i}\left|v^{*}\right|_{i, \mathbb{K}}^{2} \leqq & \left.v^{*}\right|_{0, \mathrm{R}} ^{2}+c\left|v^{*}\right|_{1, \mathrm{~K}}^{2} h^{-2 k} \\
& \leqq c h^{-n}\left(\|v\|_{0, K}^{2}+h^{-2 k+2}\|v\|_{1, K}^{2}\right)
\end{aligned}
$$

Hence

$$
\sum_{i=0}^{k} h^{-2 i}\left|v^{*}\right|_{i, K}^{2} \leqq c h^{-n-2 k+2}\|v\|_{1, K}^{2} \quad \text { for } \quad k \geqq 1 .
$$

Similarly

$$
\sum_{i=0}^{k} h^{-2 i}\left|v^{*}\right|_{i, K}^{2} \leqq c h^{-n-2 k+4}\|v\|_{2, K}^{2} \quad \text { for } \quad k \geqq 2 .
$$

Substituting from (2.44) into (2.42) and observing that $m \geqq k$ and $d \geqq 2 k-2$, we obtain

$$
\left|\hat{E}\left(J_{K} \varphi^{*} v^{*}\right)\right|^{2} \leqq c h^{2 k}\|\varphi\|_{m, K}^{2}\|v\|_{1, K}^{2} \quad \text { for } \quad k \geqq 1 .
$$

Substituting from (2.45) into (2.43) and observing that $d \geqq 2 k-2$, we get

$$
\left|\hat{E}\left(J_{K} \varphi^{*} v^{*}\right)\right|^{2} \leqq c h^{2(k+1)}\|\varphi\|_{k+1, K}^{2}\|v\|_{2, K}^{2} \quad \text { for } \quad k \geqq 2 .
$$

Substituting from (2.44) into (2.43) and observing that $d \geqq 1$, we obtain

$$
\left|\hat{E}\left(J_{K} \varphi^{*} v^{*}\right)\right|^{2} \leqq c h^{4}\|\varphi\|_{2, K}^{2}\|v\|_{1, K}^{2} \quad \text { for } \quad k=1 .
$$


From (2.47) and (2.48) we see that

$$
\left|\hat{E}\left(J_{K} \varphi^{*} v^{*}\right)\right|^{2} \leqq c h^{2(k+1)}\|\varphi\|_{k+1, K}^{2}\|v\|_{2, K}^{2} \quad \text { for } \quad k \geqq 1 .
$$

From (2.46), (2.49), (2.41) and from the Schwarz inequality the inequalities (2.39) and (2.40) follow.

THEOREM 2.2: Let $\mathscr{C}_{h}$ be a k-regular triangulation of the set $\Omega$, where $k>n / 2-1$. Let $\varphi \in V_{h}(\Omega), v \in V_{h}(\Omega), b \in C^{k+1}\left(\bar{\Omega}_{h}\right)$ and $\Phi \in H^{k+1}\left(\Omega_{h}\right)$ be any function such that $\pi_{h} \Phi \in V_{h}(\Omega)$. Let the quadrature formula given on the reference set $\hat{K}$ be of a degree $d \geqq \max (1,2 k-2)$.

Then there exists a constant $c$ such that

$$
\left|E\left(b \frac{\partial \varphi}{\partial x_{i}} \frac{\partial v}{\partial x_{j}}\right)\right| \leqq c\left[h^{k}\|\Phi\|_{k+1, \Omega_{n}}+\|\varphi-\Phi\|_{0, \Omega_{n}}\right]\|v\|_{1, \Omega_{n}} .
$$

If, in addition, $b \in C^{k+2}\left(\bar{\Omega}_{h}\right)$ then there exists a constant $c$ such that

$$
\left|E\left(b \frac{\partial \varphi}{\partial x_{i}} \frac{\partial v}{\partial x_{j}}\right)\right| \leqq c h\left[h^{k}\|\Phi\|_{k+1, \Omega_{n}}+\|\varphi-\Phi\|_{0, \Omega_{n}}\right]\left(\sum_{K \in \mathscr{Q}_{n}}\|v\|_{2, K}^{2}\right)^{1 / 2}
$$

Proof: Obviously

$$
E\left(b \frac{\partial \varphi}{\partial x_{i}} \frac{\partial v}{\partial x_{j}}\right)=\sum_{K \in \mathscr{\vartheta}_{i}} \hat{E}\left(J_{K} b^{*}\left(\frac{\partial \varphi}{\partial x_{i}}\right)^{*}\left(\frac{\partial v}{\partial x_{j}}\right)^{*}\right) .
$$

From the rule on differentiation of the composite function it follows

$$
\hat{E}\left(J_{K} b^{*}\left(\frac{\partial \varphi}{\partial x_{i}}\right)^{*}\left(\frac{\partial v}{\partial x_{j}}\right)^{*}\right)=\sum_{r, p=1}^{n} \hat{E}\left(\gamma_{i, j} \frac{\partial \varphi^{*}}{\partial \hat{x}_{r}} \frac{\partial v^{*}}{\partial \hat{x}_{p}}\right)
$$

where

$$
\gamma_{i, j}=b^{*} \frac{J_{K}^{(r, i)} J_{K}^{(p, j)}}{J_{K}} \quad\left(J_{K}^{(r, i)}, J_{K}^{(p, j)} \text { are cofactors of } J_{K}\right) .
$$

From lemma 2.3 and from lemma 2.1 we get $D^{\alpha}\left(\gamma_{i j}\right)=O\left(h^{|\alpha|+n-2}\right)$. Hence we may apply lemma 2.6 for $\psi=\varphi^{*}, s=k+q\left(q=1\right.$ if $b \in C^{k+1}$ or $q=2$ if $\left.b \in C^{k+2}\right)$, $\tau=v^{*}, r=k, \delta=\gamma_{i j}, \mathscr{H}=n-2$ and $d \geqq \max (1,2 k-2)$. From (2.23) we get

$$
\begin{aligned}
\left|\hat{E}\left(\gamma_{i j} \frac{\partial \varphi^{*}}{\partial \hat{x}_{i}} \frac{\partial v^{*}}{\partial \hat{x}_{j}}\right)\right|^{2} \leqq & c h^{2(n-2)}\left\{h^{2(k+q)}\left\|\varphi^{*}\right\|_{0, \mathrm{~K}}^{2}\left|v^{*}\right|_{1, \mathrm{R}}^{2}\right. \\
& \left.+h^{2(d+3)} \sum_{i=1}^{k} h^{-2 i}\left|\varphi^{*}\right|_{i, \mathrm{R}}^{2} \sum_{i=1}^{k} h^{-2 i}\left|v^{*}\right|_{i, \mathrm{R}}^{2}\right\} .
\end{aligned}
$$

vol. $13, n^{\circ} 3,1979$ 
From lemma 2.2, lemma 2.4 and from the interpolation theorem (see lemma 1.1) it follows

$$
\begin{aligned}
& \sum_{i=1}^{k} h^{-2 i}\left|\varphi^{*}\right|_{i, \mathrm{~K}}^{2} \\
& \leqq c\left\{\sum_{i=1}^{k} h^{-2 i}\left|\varphi^{*}-\left(\pi_{h} \Phi\right)^{*}\right|_{i, K}^{2}+\sum_{i=1}^{k} h^{-2 i}\left|\left(\pi_{h} \Phi\right)^{*}-\Phi^{*}\right|_{i, K}^{2}+\sum_{i=1}^{k} h^{-2 i}\left|\Phi^{*}\right|_{i, K}^{2}\right\} \\
& \leqq c h^{-n}\left\{h^{-2 k}\left\|\varphi-\pi_{h} \Phi\right\|_{0, K}^{2}+\sum_{i=1}^{k}\left\|\pi_{h} \Phi-\Phi\right\|_{i, K}^{2}+\sum_{i=1}^{k}\|\Phi\|_{i, K}^{2}\right\} \\
& \leqq c h^{-n}\left\{h^{-2 k}\left(\|\varphi-\Phi\|_{0, K}^{2}+\left\|\Phi-\pi_{h} \Phi\right\|_{0, K}^{2}\right)+\left\|\pi_{h} \Phi-\Phi\right\|_{k, K}^{2}+\|\Phi\|_{k, K}^{2}\right\} \\
& \leqq c h^{-n}\left\{h^{-2 k}\left(\|\varphi-\Phi\|_{0, K}^{2}+h^{2 k+2}\|\Phi\|_{k+1, K}^{2}\right)+h^{2}\|\Phi\|_{k, K}^{2}+\|\Phi\|_{k, K}^{2}\right\} .
\end{aligned}
$$

Hence

$$
\sum_{i=1}^{k} h^{-2 i}\left|\varphi^{*}\right|_{i, K}^{2} \leqq c h^{-n}\left\{h^{-2 k}\|\varphi-\Phi\|_{0, K}^{2}+\|\Phi\|_{k+1, K}^{2}\right\} .
$$

Evidently

$$
\left\|\varphi^{*}\right\|_{0, K}^{2} \leqq c h^{-n}\|\varphi\|_{0, K}^{2}, \quad\left|v^{*}\right|_{1, R}^{2} \leqq c h^{-n+2}\|v\|_{1, K}^{2} .
$$

Substituting from (2.56), (2.55) and from (2.44) into (2.54) for $q=1$ and observing that $d \geqq 2 k-2$ we get (for $k \geqq 1$ ):

$$
\begin{aligned}
&\left|\hat{E}\left(\gamma_{i j} \frac{\partial \varphi^{*}}{\partial \hat{x}_{i}} \frac{\partial v^{*}}{\partial \hat{x}_{j}}\right)\right|^{2} \leqq c h^{2(n-2)}\left\{h^{2 k+2} h^{-n}\|\varphi\|_{0, K}^{2} h^{-n+2}\|v\|_{1, K}^{2}\right. \\
&\left.+h^{2(2 k-2+3)} h^{-n}\left(h^{-2 k}\|\varphi-\Phi\|_{0, K}^{2}+\|\Phi\|_{k+1, K}^{2}\right) h^{-n-2 k+2}\|v\|_{1, K}^{2}\right\} \\
& \leqq c\left\{h^{2 k}\left(\|\varphi-\Phi\|_{0, K}^{2}+\|\Phi\|_{0, K}^{2}\right)\|v\|_{1, K}^{2}\right. \\
&\left.\quad+h^{2 k}\left(h^{-2 k}\|\varphi-\Phi\|_{0, K}^{2}+\|\Phi\|_{k+1, K}^{2}\right)\|v\|_{1, K}^{2}\right\} .
\end{aligned}
$$

Hence$$
\left|\hat{E}\left(\gamma_{i j} \frac{\partial \varphi^{*}}{\partial \hat{x}_{i}} \frac{\partial v^{*}}{\partial \hat{x}_{j}}\right)\right|^{2} \leqq c\left[\|\varphi-\Phi\|_{0, K}^{2}+h^{2 k}\|\Phi\|_{k+1, K}^{2}\right]\|v\|_{1, K}^{2}
$$

for $k \geqq 1 . \quad(2.57)$

Substituting from (2.56), (2.55) and from (2.45) into (2.54) for $q=2$ and observing that $d \geqq 2 k-2$, we similarly obtain

$$
\begin{aligned}
\left|\hat{E}\left(\gamma_{i j} \frac{\partial \varphi^{*}}{\partial \hat{x}_{i}} \frac{\partial v^{*}}{\partial \hat{x}_{j}}\right)\right|^{2} & \\
& \leqq c h^{2}\left[\|\varphi-\Phi\|_{0, K}^{2}+h^{2 k}\|\Phi\|_{k+1, K}^{2}\right]\|v\|_{2, K}^{2} \quad \text { for } \quad k \geqq 2
\end{aligned}
$$


Substituting from (2.56), (2.55) and from (2.44) into (2.54) for $k=1, q=2$ and observing that $d \geqq 1$, we get

Hence

$$
\begin{aligned}
&\left|\hat{E}\left(\gamma_{i j} \frac{\partial \varphi^{*}}{\partial \hat{x}_{i}} \frac{\partial v^{*}}{\partial \hat{x}_{j}}\right)\right|^{2} \leqq c h^{2 n-4}\left\{h^{6} h^{-n}\|\varphi\|_{0, K}^{2} h^{-n+2}\|v\|_{1, K}^{2}\right. \\
&\left.+h^{8} h^{-n}\left(h^{-2}\|\varphi-\Phi\|_{0, K}^{2}+\|\Phi\|_{2, K}^{2}\right) h^{-n}\|v\|_{1, K}^{2}\right\} \\
& \leqq c\left\{h^{4}\left(\|\varphi-\Phi\|_{0, K}^{2}+\|\Phi\|_{0, K}^{2}\right)\|v\|_{1, K}^{2}\right. \\
&\left.+h^{4}\left(h^{-2}\|\varphi-\Phi\|_{0, K}^{2}+\|\Phi\|_{2, K}^{2}\right)\|v\|_{1, K}^{2}\right\} .
\end{aligned}
$$

$$
\left|\hat{E}\left(\gamma_{i j} \frac{\partial \varphi^{*}}{\partial \hat{x}_{i}} \frac{\partial v^{*}}{\partial \hat{x}_{j}}\right)\right|^{2} \leqq c h^{2}\left[\|\varphi-\Phi\|_{0, K}^{2}+h^{2}\|\Phi\|_{2, K}^{2}\right]\|v\|_{1, K}^{2} .
$$

From (2.58) and (2.59) we see that

$$
\begin{aligned}
\left|\hat{E}\left(\gamma_{i j} \frac{\partial \varphi^{*}}{\partial \hat{x}_{i}} \frac{\partial v^{*}}{\partial \hat{x}_{j}}\right)\right|^{2} & \\
& \leqq c h^{2}\left[\|\varphi-\Phi\|_{0, K}^{2}+h^{2 k}\|\Phi\|_{k+1, K}^{2}\right]\|v\|_{2, K}^{2} \quad \text { for } \quad k \geqq 1 .
\end{aligned}
$$

From (2.57), (2.60), (2.53), (2.52) and from the Schwarz inequality the inequalities (2.50) and (2.51) follow.

\section{APPROXIMATE SOLUTION OF THE ELLIPTIC PROBLEMS}

Let $\Omega$ be a bounded domain in $R^{n}$ with sufficiently smooth boundary $\partial \Omega$. We study the elliptic problem

$$
\left.\begin{array}{cc}
-l u=f(x), & x \in \Omega, \\
u(x)=0 & \text { on } \partial \Omega,
\end{array}\right\}
$$

where $f$ is a sufficiently smooth function and

$$
l=\sum_{i, j=1}^{n} \frac{\partial}{\partial x_{j}}\left(g_{i j}(x) \frac{\partial}{\partial x_{i}}\right) .
$$

We suppose that the functions $g_{i j}(x)$ are sufficiently smooth and

$$
g_{i j}(x)=g_{j i}(x) .
$$

About the differential operator $l$ we suppose that it is strongly elliptic, i. e. there exists a constant $g_{1}>0$ such that

$$
\sum_{i, j=1}^{n} g_{i j}(x) \xi_{i} \xi_{j} \geqq g_{1} \sum_{i=1}^{n} \xi_{i}^{2}, \quad \forall x \in \bar{\Omega}, \quad\left(\xi_{1}, \ldots, \xi_{n}\right) \in R^{n} .
$$

vol. $13, \mathrm{n}^{\circ} 3,1979$ 
The variational formulation of the elliptic problem is:

Find a function $u(x) \in H_{0}^{1}(\Omega)$ such that

$$
\left.\begin{array}{l}
\in H_{0}^{1}(\Omega) \text { such that } \\
a(u, v)=(f, v)_{0, \Omega}, \forall v \in H_{0}^{1}(\Omega),
\end{array}\right\}
$$

where

$$
a(u, v)=\int_{\Omega} \sum_{i, j=1}^{n} g_{i j}(x) \frac{\partial u}{\partial x_{i}} \frac{\partial v}{\partial x_{j}} d x
$$

We extend the functions $g_{i j}(x), f(x)$ to a greater set $\tilde{\Omega} \supset \Omega$ so that the conditions (3.3) and (3.4) are satisfied (with positive constants $G_{1}$ ). In this way we obtain the functions $G_{i j}(x), F(x)$. We denote

$$
L=\sum_{i, j=1}^{n} \frac{\partial}{\partial x_{j}}\left(G_{i j}(x) \frac{\partial}{\partial x_{i}}\right)
$$

Let $\mathscr{C}_{h}$ be a $k$-regular triangulation of the set $\Omega$ and Let $V_{h}$ be the corresponding finite element space. The union of the elements $K$ from $\mathscr{C}_{h}$ forms a set $\Omega_{h}$ which, in general, differs from $\Omega$. We suppose that

$$
\Omega_{h} \subset \tilde{\Omega},
$$

for all sufficiently small $h$ and formulate the following discrete problem

Find a function $u_{d}(x) \in V_{h}$ such that

$$
\left.\begin{array}{l}
a_{h}\left(u_{d}, v\right)=(F, v)_{\Omega, \Omega}, \quad \forall v \in V_{h},
\end{array}\right\}
$$

where

$$
a_{h}\left(u_{d}, v\right)=\int_{\Omega_{n}} \sum_{i, j=1}^{n} G_{i j}(x) \frac{\partial u}{\partial x_{i}} \frac{\partial v}{\partial x_{j}} d x .
$$

Since it is either too costly or simply impossible to evaluate exactly the integrals $(., .)_{0, \Omega_{h}}, a_{h}(.,$.$) , we must now take into account the fact that approximate$ integration is used for their computation. For this purpose we use the isoparametric numerical integration, i.e. in agreement with (2.4) we replace

$$
(\varphi, \psi)_{0, \Omega_{h}} \approx(\varphi, \psi)_{h}, \quad a_{h}(\varphi, \psi) \approx A_{h}(\varphi, \psi)
$$

where

$$
(\varphi, \psi)_{h}=\sum_{K \in \mathscr{\vartheta}_{h}} \sum_{r} \omega_{r, K} \varphi\left(b_{r, K}\right) \psi\left(b_{r, K}\right)
$$




$$
A_{h}(\varphi, \psi)=\sum_{K \in \mathscr{Q}_{n}} \sum_{r} \omega_{r, K} \sum_{i, j=1}^{n} G_{i j}\left(b_{r, K}\right) \frac{\partial \varphi}{\partial x_{i}}\left(b_{r, K}\right) \frac{\partial \psi}{\partial x_{j}}\left(b_{r, K}\right)
$$

Let us note that form (2.6) and (2.8) it follows

$$
\begin{gathered}
(\varphi, \psi)_{0, \Omega_{h}}-(\varphi, \psi)_{h}=E(\varphi \psi), \\
a_{h}(\varphi, \psi)-A_{h}(\varphi, \psi)=E\left(\sum_{i, j=1}^{n} G_{i j}(x) \frac{\partial \varphi}{\partial x_{i}} \frac{\partial \psi}{\partial x_{j}}\right) .
\end{gathered}
$$

Evidently $b_{r, K} \in \bar{\Omega}$ for sufficiently small $h$ (remember that $\hat{b}_{r}$ are supposed to lie inside $\hat{K}$ or coincide with some of the points $\left.\hat{a}_{i}\right)$. Hence $F\left(b_{r, K}\right)=f\left(b_{r, K}\right)$, $G_{i j}\left(b_{r, K}\right)=g_{i j}\left(b_{r, K}\right)$. Therefore from $(3.12)$ we see that $(F, v)_{h}=(f, v)_{h}$. In such a way we come to the following fully discrete problem:

Find a function $u_{h}(x) \in V_{h}$ such that

$$
\left.A_{h}\left(u_{h}, v\right)=(f, v)_{h}, \quad \forall v \in V_{h}\right\}
$$

Let the functions $\varphi_{1}, \ldots, \varphi_{s}$ form the basis of the space $V_{h}$. Denoting

$$
\begin{gathered}
\gamma=\left[\gamma_{1}, \ldots, \gamma_{s}\right]^{T}, \\
\mathbf{K}_{h}=\left\{A_{h}\left(\varphi_{i}, \varphi_{j}\right)\right\}_{i, j=1}^{s} \\
\mathbf{F}_{h}=\left[\left(f, \varphi_{1}\right)_{h}, \ldots,\left(f, \varphi_{s}\right)_{h}\right]^{T}
\end{gathered}
$$

the system (3.16) can be written in the form

$$
\mathbf{K}_{h} \gamma=\mathbf{F}_{h} \text {. }
$$

\section{APPROXIMATE SOLUTION OF THE PARABOLIC PROBLEMS}

We study the parabolic problem

$$
\left.\begin{array}{c}
g(x) \frac{\partial w}{\partial t}-l u=f(x, t) \quad \text { for } \quad x \in \Omega \text { and } t \in(0, T), \\
w(x, t)=0 \quad \text { for } \quad x \in \partial \Omega \text { and } t \in(0, T), \\
w(x, 0)=w_{0}(x) \in L^{2}(\Omega),
\end{array}\right\}
$$

where $g(x)$ and $f(x, t)$ are sufficiently smooth functions,

$$
g(x) \geqq g_{0}(=\text { Const. })>0
$$

vol. $13, \mathrm{n}^{\circ} 3,1979$ 
and the differential operator $l$ defined by (3.2) satisfies the conditions (3.3) and (3.4) with sufficiently smooth functions $g_{i j}(x)$. Similarly as in the elliptic case we come to the variational formulation of the parabolic problem (see [8]):

Find a function $w(x, t)$ such that

$$
\begin{gathered}
w \in L^{\infty}\left(H_{0}^{1}(\Omega)\right), \frac{\partial w}{\partial t} \in L^{\infty}\left(H^{-1}(\Omega)\right) \\
\left(g \frac{\partial w}{\partial t}, v\right)_{0, \Omega_{n}}+a(w, v)=(f, v)_{0, \Omega_{n}}, \\
\forall v \in H_{0}^{1}(\Omega) \text { and } t \in(0, T) \\
w(x, 0)=w_{0}(x) \in L^{2}(\Omega)
\end{gathered}
$$

where the bilinear form $a(.,$.$) is given by (3.6).$

Let us denote by $G(x)$ a sufficiently smooth extension of the function $g(x)$ to a greater set $\widetilde{\Omega}$ satisfying (4.2) (with some positive constant $G_{0}$ ). First, in the same way as in the elliptic case, we discretize this problem for every $t \in(0, T)$ by the finite element method with respect to $x$. Then we use isoparametric numerical integration. In such a way we come to the following fully semidiscrete problem:

Find a function $w_{s}(x, t)$ such that

$$
\left.\begin{array}{c}
w_{s}, \frac{\partial w_{s}}{\partial t} \in V_{h}, \quad \forall t \in(0, T) \\
\left(g \frac{\partial w_{s}}{\partial t}, v\right)_{h}+A_{h}\left(w_{s}, v\right)=(f, v)_{h}, \\
\forall v \in V_{h} \text { and } t \in(0, T), \\
w_{s}(x, 0)=w_{s 0}(x),
\end{array}\right\}
$$

where $w_{\mathrm{s} 0}(x)$ is an approximation of $w_{0}(x)$.

Replacing $v$ in (4.4) by the basic functions $\varphi_{i}$ we come, to the conclusion that the problem (4.4) is represented by the system of ordinary differential equations with an unknown vector function of parametre $t$ :

$$
\mathbf{M}_{h} \boldsymbol{\gamma}^{\prime}(t)+\mathbf{K}_{h} \gamma(t)=\mathbf{F}_{n}
$$

where

$$
\begin{aligned}
\gamma(t) & =\left[\gamma_{1}(t), \ldots, \gamma_{s}(t)\right]^{T}, \\
\mathbf{M}_{h} & =\left\{\left(g \varphi_{i}, \varphi_{j}\right)_{h}\right\}_{i, j=1}^{s},
\end{aligned}
$$




$$
\begin{gathered}
\mathbf{K}_{h}=\left\{A_{h}\left(\varphi_{i}, \varphi_{j}\right)\right\}_{i, j=1}^{s}, \\
\mathbf{F}_{h}(t)=\left[\left(f, \varphi_{1}\right)_{h}, \ldots,\left(f, \varphi_{s}\right)_{h}\right]^{T} .
\end{gathered}
$$

This suggests the way how to discretize the problem (4.4) with respect to $t$. We solve the mentioned system of ordinary differential equations by $v$-step $A_{0}$-stable method of order $q$. We divide the time interval $(0, T)$ into a finite number of equal parts $\Delta t$. We introduce the notation

$$
\Phi^{m}=\Phi^{m}(x)=\Phi(x, m \Delta t), \quad m=0,1, \ldots
$$

for any function $\Phi(x, t)$.

According to (4.4) and to the described way of the time discretization we define the following fully discrete problem

Find a function $w_{h}(x, t)$ such that

$$
\begin{gathered}
w_{h} \in V_{h} \quad \text { for } t=\Delta t, 2 \Delta t, \ldots, T, \\
\left(g \sum_{j=0}^{v} \alpha_{j} w_{h}^{m+j}, v\right)_{h}+\Delta t A_{h}\left(\sum_{j=0}^{v} \beta_{j} w_{h}^{m+j}, v\right) \\
=\Delta t\left(\sum_{j=0}^{v} \beta_{j} f^{m+j}, v\right)_{h}, \quad \forall v \in V_{h} \text { and } m=0,1, \ldots \\
w_{h}^{0}=w_{s 0}(x) .
\end{gathered}
$$

From (4.5) we can see that the system in (4.11) is represented by the linear system of algebraic equations

$$
\sum_{j=0}^{v}\left(\alpha_{j} \mathbf{M}_{h}+\Delta t \beta_{j} \mathbf{K}_{h}\right) \gamma^{m+j}=\Delta t \sum_{j=0}^{v} \beta_{j} \mathbf{F}_{h}^{m+j}
$$

i.e. by the system

$$
\begin{aligned}
\left(\alpha_{v} \mathbf{M}_{h}+\Delta t \beta_{v} \mathbf{K}_{h}\right) \gamma^{m+v}=\Delta t & \beta_{v} \mathbf{F}_{h}^{m+v} \\
& +\sum_{j=0}^{v-1}\left[\Delta t \beta_{j}\left(\mathbf{F}_{h}^{m+j}-\mathbf{K}_{h} \gamma^{m+j}\right)-\alpha_{j} \mathbf{M}_{h} \gamma^{m+j}\right]
\end{aligned}
$$

\section{RITZ APPROXIMATIONS}

Let $U$ be a function from $H^{1}(\widetilde{\Omega})$. The function $\eta \in V_{h}\left(\Omega_{h}\right)$ such that

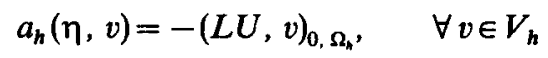

vol. $13, \mathrm{n}^{\circ} 3,1979$ 
is called the Ritz approximation of the function $U$. The function $\eta_{d} \in V_{h}(\Omega)$ such that

$$
A_{h}\left(\eta_{d}, v\right)=-(L U, v)_{h}, \quad \forall v \in \dot{V_{h}}
$$

is called the Ritz discrete approximation of the function $U$.

From the Green theorem it follows

$$
a_{h}(\eta, v)=a_{h}(U, v), \quad \forall v \in V_{h},
$$

i. e. the function $\eta$ is an orthogonal projection onto $V_{h}$ of the function $U$ in the energy norm given by the bilinear form $a_{h}(.,$.$) . This is the reson why we use the$ name Ritz approximation. From the proof of theorem 1 in [7] the following theorem follows:

TheOREM 5.1 (theorem on the Ritz approximation): Let $\mathscr{C}_{h}$ be a $k$-regular triangulation of the set $\Omega, k>n / 2-1$ and let

$$
\Omega_{h} \subset \tilde{\Omega} \text { for all } h \text {. }
$$

Let $U \in H^{k+1}(\tilde{\Omega})$ be any function such that

$$
U=0 \text { on } \partial \Omega
$$

and let $\eta$ be the Ritz approximation of the function $U$.

Then there exists a constant $c$ (independent of $h$ ) such that

$$
|u-\eta|_{1, \Omega_{n}} \leqq c h^{k}\|U\|_{k+1, \Omega_{n}} \text {. }
$$

If, in addition, $U \in H^{k+2}(\tilde{\Omega})$, then there exists a constant $c$ such that

$$
\|U-\eta\|_{0, \Omega_{n}} \leqq c h^{k+1}\|U\|_{k+2, \Omega} .
$$

Remark: From (5.6) and (5.7) it follows immediately

$$
\|U-\eta\|_{1, \Omega_{n}} \leqq c h^{k}\|U\|_{k+2, \Omega}
$$

provided $U \in H^{k+2}(\widetilde{\Omega})$.

We are going to derive the similar theorem for the Ritz discrete approximation. Before, we formulate two lemmas.

Lemma 5.1. Let $\mathscr{C}_{h}$ be a k-regular triangulation of $\Omega(k>(n / 2)-1)$. Let $v \in H^{1}(\widetilde{\Omega})$ and

$$
v\left(y^{\prime}, y_{n}\right)=0 \quad \text { on } \partial \Omega_{h}
$$

R.A.I.R.O. Analyse numérique/Numerical Analysis 
(for notation see figure). Then there exists a constant $c$ such that

$$
\|v\|_{0, \Omega_{n}-\Omega} \leqq c h^{k+1}|v|_{1, \Omega_{n}-\Omega} .
$$

The proof follows from [7] (see lemma 1 and note 1).

We introduce the notation

$$
\|v\|_{h}^{2}=(g(x) v, v)_{h}, \quad|v|_{h}^{2}=A_{h}(v, v),
$$

where the forms $(., .)_{h}, \mathrm{~A}_{h}(.,$.$) are defined in (3.12) and (3.13).$

LEMMA 5.2. Let $\mathscr{C}_{h}$ be a $k$-regular triangulation of $\Omega(k>(n / 2)-1)$. Then there exist positive constants $c_{1}$ and $c_{2}$ such that:

$$
c_{1}\|v\|_{0, \Omega_{n}} \leqq\|v\|_{h}, \quad \forall v \in V_{h}
$$

provided the quadrature formula on the refrence set $\hat{K}$ is of a degree $d \geqq 2 k$,

$$
c_{2}|v|_{1, \Omega_{n}} \leqq|v|_{h}, \quad \forall v \in V_{h}
$$

provided the quadrature formula on the reference set $\hat{K}$ is of a degree $d \geqq 2 k-2$.

For the proof see [7] (Theorem 5).

THEOREM 5.2 (Theorem on the Ritz discrete approximation): Let $\mathscr{C}_{h}$ be a $k$-regular triangulation of the set $\Omega, k>(n / 2)-1,(5.4)$ be satisfied and $h<1$. Let $U \in H^{m+2}(\tilde{\Omega})$, where $m=\max ([n / 2]+1, k)$ be any function such that $U=0$ on $\partial \Omega$ and the quadrature formula given on the reference set $\hat{K}$ be of a degree $d \geqq \max (1,2 k-2)$. Let $\eta_{d}$ be the Ritz discrete approximation of the function $U$. Then there exists a constant $c$ such that

$$
\left\|U-\eta_{d}\right\|_{1, \Omega_{n}} \leqq c h^{k}\|U\|_{m+2, \widetilde{\Omega}} .
$$

If, in addition, $U \in H^{k+3}(\tilde{\Omega})$ then there exist constants $c_{1}, c_{2}$ such that

$$
\begin{gathered}
\left\|U-\eta_{d}\right\|_{0, \Omega_{n}} \leqq c h^{k+1}\|U\|_{k+3, \tilde{\Omega}}, \\
\left\|U-\eta_{d}\right\|_{h} \leqq c h^{k+1}\|U\|_{k+3, \tilde{\Omega}} .
\end{gathered}
$$

Proof: Evidently

$$
\left|U-\eta_{d}\right|_{1, \Omega_{n}} \leqq|U-\eta|_{1, \Omega_{n}}+\left|\eta-\eta_{d}\right|_{1, \Omega_{n}},
$$

where $\eta$ is the Ritz approximation of the function $U$.

From (5.13) (lemma 5.2 may be applied since $\eta-\eta_{d} \in V_{h}$ ), from (3.15), (5.1), (5.2) and from (3.14) it follows

$$
\begin{aligned}
& \left|\eta-\eta_{d}\right|_{1, \Omega_{n}}^{2} \leqq c A_{h}\left(\eta-\eta_{d}, \eta-\eta_{d}\right)=c\left\{A_{h}\left(\eta, \eta-\eta_{d}\right)-A_{h}\left(\eta_{d}, \eta-\eta_{d}\right)\right\} \\
& \quad=c\left\{a_{h}\left(\eta, \eta-\eta_{d}\right)-E\left(\sum_{i, j=1}^{n} G_{i j} \frac{\partial \eta}{\partial x_{i}} \frac{\partial\left(\eta-\eta_{d}\right)}{\partial x_{j}}\right)-A_{h}\left(\eta_{d}, \eta-\eta_{d}\right)\right\}
\end{aligned}
$$

vol. $13, n^{\circ} 3,1979$ 


$$
\begin{aligned}
=c\left\{-\left(L U, \eta-\eta_{d}\right)_{0, \Omega_{n}}-E\left(\sum_{i, j=1}^{n} G_{i j} \frac{\partial \eta}{\partial x_{i}} \frac{\partial\left(\eta-\eta_{d}\right)}{\partial x_{j}}\right)+\left(L U, \eta-\eta_{d}\right)_{h}\right\} \\
=c\left\{-E\left(L U\left(\eta-\eta_{d}\right)\right)-E\left(\sum_{i, j=1}^{n} G_{i j} \frac{\partial \eta}{\partial x_{i}} \frac{\partial\left(\eta-\eta_{d}\right)}{\partial x_{j}}\right)\right\} .
\end{aligned}
$$

Hence, using the inequality (2.39) for $\varphi=L U, v=\eta-\eta_{d}$ and the inequality (2.50) for $\varphi=\eta, v=\eta-\eta_{d}$ and $\Phi=U$ (notice that $\pi_{h} U \in V_{h}$ ) we get

$$
\begin{aligned}
&\left|\eta-\eta_{d}\right|_{1, \Omega_{n}}^{2} \leqq c\left\{h^{k}\|L U\|_{m, \Omega_{n}}+h^{k}\|U\|_{k+1, \Omega_{n}}\right. \\
&\left.+\|\eta-U\|_{0, \Omega_{n}}\right\}\left\|\eta-\eta_{d}\right\|_{1, \Omega_{n}} .
\end{aligned}
$$

We notice at this point that because of the assumption (5.4) there exists a constant $c$ independent of $h$ such that

$$
\|v\|_{1, \Omega_{n}} \leqq c|v|_{1, \Omega_{n^{\prime}}} \quad \forall v \in V_{h}
$$

(see Ciarlet and Raviart [3], p. 455).

Therefore from (5.18) and from the theorem on the Ritz approximation [see (5.7)] it follows

$$
\left|\eta-\eta_{d}\right|_{1, \Omega_{n}}^{2} \leqq c h^{k}\|U\|_{m+2, \Omega}\left|\eta-\eta_{d}\right|_{1, \Omega_{n}} .
$$

Hence

$$
\left|\eta-\eta_{d}\right|_{1, \Omega_{k}} \leqq c h^{k}\|U\|_{m+2, \Omega}
$$

From (5.17), (5.6) and (5.20) we get

$$
\left|U-\eta_{d}\right|_{1, \Omega_{k}} \leqq c h^{k}\|U\|_{m+2, \Omega} .
$$

Evidently

$$
\left\|U-\eta_{d}\right\|_{0, \Omega_{n}} \leqq\|U-\eta\|_{0, \Omega_{k}}+\left\|\eta-\eta_{d}\right\|_{0, \Omega_{k}} .
$$

Therefore from the theorem on the Ritz approximation, from (5.19) and from (5.20) it follows

$$
\left|U-\eta_{d}\right|_{0, \Omega_{m}} \leqq c h^{k}\|U\|_{m+2, \Omega} .
$$

The inequalities (5.21) and (5.22) imply (5.14).

We prove now the inequality (5.15). We give the proof for $n \leqq 3$; the proof for $n>3$ can be achieved by using a smoothing procedure, following an idea of Strang [9]. 
Let us denote

$$
z=\left\{\begin{array}{lll}
\eta-\eta_{d} & \text { for } & x \in \bar{\Omega}_{h} \\
0 & \text { for } & x \in \tilde{\Omega}-\bar{\Omega}_{h} .
\end{array}\right\}
$$

Let $y$ be the solution of the homogeneous Dirichlet problem

$$
-l y=z \text { in } \Omega, \quad y=0 \text { on } \partial \Omega .
$$

If $\partial \Omega$ is smooth enough then $y \in H_{0}^{1}(\Omega) \cap H^{2}(\Omega)$ and

i. e. :

$$
\|y\|_{2, \Omega} \leqq c\|z\|_{0, \Omega} \leqq c\|z\|_{0 . \Omega}=c\|z\|_{0, \Omega_{n}} .
$$

$$
\|y\|_{2, \Omega} \leqq c\left\|\eta-\eta_{d}\right\|_{0, \Omega} .
$$

Using the Calderon theorem we extend the function $y$ from $\Omega$ onto $\widetilde{\Omega}$. In this way we obtain a function $\tilde{y} \in H^{2}(\tilde{\Omega})$ such that $\|\tilde{y}\|_{2, \Omega} \leqq c\|y\|_{2, \Omega}$. Therefore from (5.25) it follows

$$
\|\tilde{y}\|_{2, \Omega_{n}} \leqq c\left\|\eta-\eta_{d}\right\|_{0, \Omega_{n}}
$$

Using simple calculation we get

$$
\left\|\eta-\eta_{d}\right\|_{0, \Omega_{n}}^{2}=\int_{\Omega_{\alpha}-\Omega}\left(\eta-\eta_{d}\right)(z+L \tilde{y}) d x-\int_{\Omega_{n}}\left(\eta-\eta_{d}\right) L \tilde{y} d x .
$$

The Green theorem $\left(\eta-\eta_{d}=0\right.$ on $\left.\partial \Omega_{h}\right)$ yields

$$
-\int_{\Omega_{h}}\left(\eta-\eta_{d}\right) L \tilde{y} d x=a_{h}\left(\eta-\eta_{d}, \tilde{y}\right)
$$

Hence

$$
\left\|\eta-\eta_{d}\right\|_{0, \Omega_{n}}^{2} \leqq\left|\int_{\Omega_{n}-\Omega}\left(\eta-\eta_{d}\right)(z+L \tilde{y}) d x\right|+\left|a_{h}\left(\eta-\eta_{d}, \tilde{y}\right)\right| .
$$

The Schwarz inequality gives

$$
\left|\int_{\Omega_{n}-\Omega}\left(\eta-\eta_{d}\right)(z+L \tilde{y}) d x\right| \leqq\left\|\eta-\eta_{d}\right\|_{0, \Omega_{n}-\Omega}\|z+L \tilde{y}\|_{0, \Omega_{n}-\Omega} .
$$

Using (5.26) we get

$$
\begin{aligned}
\|z+L \tilde{y}\|_{0, \Omega_{n}-\Omega} \leqq\|z\|_{0, \Omega_{n}} & +\|L \tilde{y}\|_{0, \Omega_{k}} \\
& \leqq\left\|\eta-\eta_{d}\right\|_{0, \Omega_{n}}+c\|\tilde{y}\|_{2, \Omega_{n}} \leqq c\left\|\eta-\eta_{d}\right\|_{0, \Omega_{n}} .
\end{aligned}
$$

vol. $13, \mathrm{n}^{\circ} 3,1979$ 
From (5.10) and (5.20) it follows

$$
\left\|\eta-\eta_{d}\right\|_{0, \Omega_{n}-\Omega} \leqq c h^{k+1}\left|\eta-\eta_{d}\right|_{1, \Omega_{n}-\Omega} \leqq c h^{2 k+1}\|U\|_{m+2, \tilde{\Omega}} .
$$

Therefore from (5.29) and (5.28) we get

$$
\left|\int_{\Omega_{n}-\Omega}\left(\eta-\eta_{d}\right)(z+L \tilde{y}) d x\right| \leqq c h^{2 k+1}\|U\|_{m+2, \Omega}\left\|\eta-\eta_{d}\right\|_{0, \Omega_{n}} .
$$

Evidently

$$
\begin{aligned}
a_{h}\left(\eta-\eta_{d}, \tilde{y}\right)=a_{h}\left(\eta-U, \tilde{y}-\pi_{h} \tilde{y}\right)+a_{h}(\eta & \left.-U, \pi_{h} \tilde{y}\right) \\
& +a_{h}\left(U-\eta_{d}, \tilde{y}-\pi_{h} \tilde{y}\right)+a_{h}\left(U-\eta_{d}, \pi_{h} \tilde{y}\right) .
\end{aligned}
$$

From (5.3) (we know that $\left.\pi_{h} \tilde{y} \in V_{h}\right)$ it follows that $a_{h}\left(\eta-U, \pi_{h} \tilde{y}\right)=0$. Hence $\left|a_{h}\left(\eta-\eta_{d}, \tilde{y}\right)\right| \leqq\left|a_{h}\left(\eta-U, \tilde{y}-\pi_{h} \tilde{y}\right)\right|$

$$
+\left|a_{h}\left(U-\eta_{d}, \tilde{y}-\pi_{h} \tilde{y}\right)\right|+\left|a_{h}\left(U-\eta_{d}, \pi_{h} \tilde{y}\right)\right| .
$$

From the Schwarz inequality, from (5.6) and from the interpolation theorem (see Lemma 1) we get

$$
\left|a_{h}\left(\eta-U, \tilde{y}-\pi_{h} \tilde{y}\right)\right| \leqq c|\eta-U|_{1, \Omega_{n}}\left|\tilde{y}-\pi_{h} \tilde{y}\right|_{1, \Omega_{h}} \leqq c h^{k}\|U\|_{k+1, \Omega_{h}} h\|\tilde{y}\|_{2, \Omega_{h}} .
$$

This and (5.26) imply

$$
\left|a_{h}\left(\eta-U, \tilde{y}-\pi_{h} \tilde{y}\right)\right| \leqq c h^{k+1}\|U\|_{k+1, \Omega_{h}}\left\|\eta-\eta_{d}\right\|_{0 \Omega_{h}} .
$$

Similarly, using (5.21), we get

$$
\left|a_{h}\left(U-\eta_{d}, \tilde{y}-\pi_{h} \tilde{y}\right)\right| \leqq c h^{k+1}\left\|U \ddot{\|}_{m+2, \tilde{\Omega}} \ddot{\|} \eta-\eta_{d}\right\|_{0, \Omega_{h}} .
$$

From the Green theorem, from (3.15), (5.2) and from (3.14) we get

$$
\begin{aligned}
& \begin{array}{r}
a_{h}\left(U-\eta_{d}, \pi_{h} \tilde{y}\right)=a_{h}\left(U, \pi_{h} \tilde{y}\right)-a_{h}\left(\eta_{d}, \pi_{h} \tilde{y}\right) \\
=-\left(L U, \pi_{h} \tilde{y}\right)_{0, \Omega_{h}}-A_{h}\left(\eta_{d}, \pi_{h} \tilde{y}\right)-E\left(\sum_{i, j=1}^{n} G_{i j} \frac{\partial \eta_{d}}{\partial x_{i}} \frac{\partial \pi_{h} \tilde{y}}{\partial x_{j}}\right) \\
=-\left(L U, \pi_{h} \tilde{y}\right)_{0, \Omega_{h}}+\left(L U, \pi_{h} \tilde{y}\right)_{h}-E\left(\sum_{i, j=1}^{n} G_{i j} \frac{\partial \eta_{d}}{\partial x_{i}} \frac{\partial \pi_{h} y}{\partial x_{j}}\right) \\
=-E\left(L U \pi_{h} \tilde{y}\right)-E\left(\sum_{i, j=1}^{n} G_{i j} \frac{\partial \eta_{d}}{\partial x_{i}} \frac{\partial \pi_{h} \tilde{y}}{\partial x_{j}}\right) .
\end{array} \\
& \text { Hence }
\end{aligned}
$$

$$
\left|a_{h}\left(U-\eta_{d}, \pi_{h} \tilde{y}\right)\right| \leqq\left|E\left(L U \pi_{h} \tilde{y}\right)\right|+\left|E\left(\sum_{i, j=1}^{n} G_{i j} \frac{\partial \eta_{d}}{\partial x_{i}} \frac{\partial \pi_{h} \tilde{y}}{\partial x_{j}}\right)\right|
$$


Evidently for $K \in \mathscr{C}_{h}\left\|\pi_{h} \tilde{y}\right\|_{2, K} \leqq\left\|\pi_{h} \tilde{y}-\tilde{y}\right\|_{2, K}+\|\tilde{y}\|_{2, K}$. Hence the interpolation theorem implies $\left\|\pi_{h} \tilde{y}\right\|_{2, K} \leqq c\|\tilde{y}\|_{2, K}$ and from (5.26) we get

$$
\left(\sum_{K \in \mathscr{Q}_{h}}\left\|\pi_{h} \tilde{y}\right\|_{2, K}^{2}\right)^{1 / 2} \leqq c\left\|\eta-\eta_{d}\right\|_{0, \Omega_{h}}
$$

Therefore from (2.40) (we apply theorem 2.1 for $\varphi=L U$ and $v=\pi_{h} \tilde{y}$ ) it follows

$$
\left|E\left(L U \pi_{h} \tilde{y}\right)\right| \leqq c h^{k+1}\|L U\|_{k+1, \Omega_{h}}\left\|\eta-\eta_{d}\right\|_{0, \Omega_{h}} .
$$

Hence

$$
\left|E\left(L U \pi_{h} \tilde{y}\right)\right| \leqq c h^{k+1}\|U\|_{k+3, \Omega_{n}}\left\|\eta-\eta_{d}\right\|_{0, \Omega_{n}} .
$$

From (2.51) (we apply theorem 2.2 for $b=G_{i j}, \varphi=\eta_{d}, \Phi=U, v=\pi_{h} \tilde{y}$ ) and from (5.35) it follows

$$
\left|E\left(\sum_{i, j=1}^{n} G_{i j} \frac{\partial \eta_{d}}{\partial x_{i}} \frac{\partial \pi_{h} \tilde{y}}{\partial x_{j}}\right)\right| \leqq c h\left[h^{k}\|U\|_{k+1, \Omega_{h}}+\left\|\eta_{d}-U\right\|_{0, \Omega_{h}}\right]\left\|\eta-\eta_{d}\right\|_{0, \Omega_{h}} .
$$

Hence, from (5.22) observing that $m \leqq k+1$ we get

$$
\left|E\left(\sum_{i, j=1}^{n} G_{i j} \frac{\partial \eta_{d}}{\partial x_{i}} \frac{\partial \pi_{h} \tilde{y}}{\partial x_{j}}\right)\right| \leqq c h^{k+1}\|U\|_{k+3, \Omega}\left\|\eta-\eta_{d}\right\|_{0, \Omega_{h}} .
$$

From (5.34), (5.36) and (5.37) it follows

$$
\left|a_{h}\left(U-\eta_{d}, \pi_{h} \tilde{y}\right)\right| \leqq c h^{k+1}\|U\|_{k+3, \Omega}\left\|\eta-\eta_{d}\right\|_{0, \Omega_{h}}
$$

Substituting from (5.32), (5.33) and (5.38) into (5.31) we get

$$
\left|a_{h}\left(\eta-\eta_{d}, \tilde{y}\right)\right| \leqq c h^{k+1}\|U\|_{k+3, \tilde{\Omega}}\left\|\eta-\eta_{d}\right\|_{0, \Omega_{h}} .
$$

From (5.27), (5.30) and (5.39) it follows

$$
\left\|\eta-\eta_{d}\right\|_{0, \Omega_{h}}^{2} \leqq c h^{k+1}\|U\|_{k+3, \Omega}\left\|\eta-\eta_{d}\right\|_{0, \Omega_{n}} .
$$

Hence

$$
\left\|\eta-\eta_{d}\right\|_{0, \Omega_{h}} \leqq c h^{k+1}\|U\|_{k+3, \Omega}
$$

Therefore, from the trivial inequality

$$
\left\|U-\eta_{d}\right\|_{0, \Omega_{n}} \leqq\|U-\eta\|_{0, \Omega_{n}}+\left\|\eta-\eta_{d}\right\|_{0, \Omega_{n}}
$$

and from the Theorem on the Ritz approximation the inequality (5.15) follows. vol. $13, \mathrm{n}^{\circ} 3,1979$ 
From (5.11), (3.12) and from (1.2) it follows

$$
\begin{aligned}
\left\|U-\eta_{d}\right\|_{h}^{2}=\sum_{K \in \mathscr{Q}_{h}} \sum_{r} \hat{\omega}_{r} J_{K}\left(\hat{b}_{r}\right) g^{*}\left(\hat{b}_{r}\right)\left[U^{*}\left(\hat{b}_{r}\right)-\eta_{d}^{*}\left(\hat{b}_{r}\right)\right]^{2} \\
\\
\quad \leqq c h^{n} \sum_{K \in \mathscr{Q}_{h}} \sum_{r}\left[U^{*}\left(\hat{b}_{r}\right)-\eta_{d}^{*}\left(\hat{b}_{r}\right)\right]^{2} .
\end{aligned}
$$

Hence

$$
\left\|U-\eta_{d}\right\|_{h}^{2} \leqq c h^{n} \sum_{K \in \mathscr{C}_{h}}\left[\max _{\mathbb{K}}\left(\mathrm{U}^{*}-\eta_{d}^{*}\right)\right]^{2}
$$

Evidently

$$
\max _{\hat{K}}\left|U^{*}-\eta_{d}^{*}\right| \leqq \max _{\hat{K}}\left|U^{*}-\pi_{k} U^{*}\right|+\max _{\hat{K}}\left|\pi_{k} U^{*}-\eta_{d}^{*}\right| .
$$

From the Bramble-Hilbert lemma and from lemma 2.4 [see (2.17)] it follows

$$
\max _{\mathbb{R}}\left|U^{*}-\pi_{k} U^{*}\right| \leqq c\left|U^{*}\right|_{k+1, \mathbb{R}} \leqq c h^{-(n / 2)+k+1}\|U\|_{k+1, K} .
$$

From lemma 2.2 [see (2.10)], from lemma 2.5 [see (2.17)], from lemma 2.4 and from the evident inequality $\left\|U^{*}-\eta_{d}^{*}\right\|_{0, K} \leqq c h^{-n / 2}\left\|U-\eta_{d}\right\|_{0, K}$ we get

$$
\begin{aligned}
\max _{\mathbb{R}}\left|\pi_{k} U^{*}-\eta_{d}^{*}\right| \leqq & \left\|\pi_{k} U^{*}-\eta_{d}^{*}\right\|_{0, \mathbb{R}} \\
\leqq & c\left[\left\|\pi_{k} U^{*}-U^{*}\right\|_{0, \mathbb{R}}+\left\|U^{*}-\eta_{d}^{*}\right\|_{0, K}\right] \\
& \leqq c\left[\left|U^{*}\right|_{k+1, \widehat{K}}+\left\|U^{*}-\eta_{d}^{*}\right\|_{0, K}\right] \\
& \leqq c h^{-n / 2}\left[h^{k+1}\|U\|_{k+1, K}+\left\|U-\eta_{d}\right\|_{0, \tilde{K}}\right] .
\end{aligned}
$$

Hence, from (5.43) and (5.42) it follows

$$
\max _{\mathbb{R}}\left|U^{*}-\eta_{d}^{*}\right| \leqq c h^{-n / 2}\left[h^{k+1}\|U\|_{k+1, K}+\left\|U-\eta_{d}\right\|_{0, K}\right] .
$$

Therefore, (5.41) implies

$$
\begin{aligned}
\left\|U-\eta_{d}\right\|_{h}^{2} \leqq c \sum_{K \in \mathscr{Q}_{h}}\left[h^{2(k+1)}\|U\|_{k+1, K}^{2}+\right. & \left.\left\|U-\eta_{d}\right\|_{0, K}^{2}\right] \\
& =c\left[h^{2(k+1)}\|U\|_{k+1, \Omega_{h}}^{2}+\left\|U-\eta_{d}\right\|_{0, \Omega_{h}}^{2}\right] .
\end{aligned}
$$

This and (5.15) imply (5.16).

\section{ERROR ESTIMATE FOR ELLIPTIC PROBLEMS}

THEOREM 6.1: Let $u$ be the solution of the elliptic problem (3.1) with sufficiently smooth functions $f, g_{i j}$ satisfying the conditions (3.3) and (3.4). Let $\mathscr{C}_{h}$ be a 
$k$-regular $(k>(n / 2)-1)$ triangulation of the set $\Omega$ with a sufficiently smooth boundary $\partial \Omega$. Let the quadrature formula on the reference set $\hat{K}$ be of a degree $d \geqq \max (1,2 k-2)$. Then the fully discrete problem (3.16) has a unique solution $u_{h}(x)$ and there exists a constant $c$ independent of $h$ and $u$ such that

$$
\begin{gathered}
\left\|u-u_{h}\right\|_{1, \Omega \cap \Omega_{k}} \leqq c h^{k}\|u\|_{m+2, \Omega}, \\
\left\|u-u_{h}\right\|_{0, \Omega \cap \Omega_{h}} \leqq c h^{k+1}\|u\|_{k+3, \Omega},
\end{gathered}
$$

where $m=\max ([n / 2]+1, k)$.

Proof: We know that the problem (3.16) is represented by the system (3.20). Hence, to prove the existence and the uniqueness it suffices to show that the matrix $\mathbf{K}_{h}$ defined by (3.18) is positive definite, i.e. that

$$
\mathbf{m}^{T} \mathbf{K}_{h} \mathbf{m}>0
$$

for any nonzero vector $\mathbf{m}=\left[m_{1}, \ldots, m_{s}\right]^{T}$.

From (3.18), (5.11) and (5.13) (we may apply lemma 5.2 since $\left.\sum_{i=1}^{s} \mathrm{~m}_{i} \varphi_{i} \in \mathrm{V}_{h}\right)$ it follows

$$
\mathbf{m}^{T} \mathbf{K}_{h} \mathbf{m}=A_{h}\left(\sum_{i=1}^{s} m_{i} \varphi_{i}, \sum_{i=1}^{s} m_{i} \varphi_{i}\right)=\left|\sum_{i=1}^{s} m_{i} \varphi_{i}\right|_{h}^{2} \geqq c\left|\sum_{i=1}^{s} m_{i} \varphi_{i}\right|_{1, \Omega_{h}}^{2}>0
$$

and $(6.3)$ is proved.

Let us suppose that $u \in H^{m+2}(\Omega)$. By the Calderon theorem there exists an extension $\tilde{u}$ of the function $u$ onto $\tilde{\Omega}$ such that

$$
\|\tilde{u}\|_{m+2, \Omega} \leqq c\|u\|_{m+2, \Omega} .
$$

Let us denote

$$
\tilde{f}=-L \tilde{u}
$$

Evidently the function $\tilde{f}$ is an extension of the function $f$.

Hence

$$
(f, v)_{h}=(\tilde{f}, v)_{h}=-(L \tilde{u}, v)_{h}
$$

Substituting from (6.6) into (3.16) we get

$$
A_{h}\left(u_{h}, v\right)=-(L \tilde{u}, v), \quad \forall v \in V_{h} .
$$

Therefore from (5.2) we can see that the function $u_{h}$ is the Ritz discrete approximation of the function $\tilde{u}$. Since $\tilde{u}=u=0$ on $\partial \Omega$ we may apply the vol. $13, \mathrm{n}^{\circ} 3,1979$ 
theorem on the Ritz discrete approximation for $\eta_{d}=u_{h}$ and $U=\tilde{u}$. From (5.14), (5.15) and (6.4) we get the estimates (6.1) and (6.2).

Remark: The results formulated in theorem 6.1 represent a generalization of the results which have been obtained for $H^{1}$ norm by Ciarlet [4] and by Zlámal [12] for special cases. In the case of selfadjoint operator $l$ they also improve the results which have been obtained for $H^{1}$ and $L_{2}$ norm by Ciarlet and Raviart [3]. They are similar to those obtained by Ženisěk [15] for $C^{m}$-elements.

\section{ERROR ESTIMATE FOR PARABOLIC PROBLEMS}

THeOREM 7.1: Let $w$ be the solution of the parabolic problem (4.1) with sufficiently smooth functions $f, g_{i j}, g$ satisfying the conditions (3.3), (3.4) and (4.2). Let $\mathscr{C}_{h}$ be a $k$-regular ( $k$ is a positive integer such that $k>n / 2-1$ ) triangulation of the set $\Omega$ with a sufficiently smooth boundary $\partial \Omega$. Let the quadrature formula on the reference set $\hat{K}$ be of a degree $d \geqq \max (1,2 k-2)$ and let exist a positive constant $c_{1}$ independent of $v$ and $h$ such that

$$
c_{1}\|v\|_{0, \Omega_{h}}^{2} \leqq(g(x) v, v)_{h}, \quad \forall v \in V_{h} .
$$

Let a given $v$-step time discretization method $(\rho, \sigma)$ of an order $q(\geqq 1)$ be $A_{0}$-stable. Besides $A_{0}$-stability, we require that the method $(\rho, \sigma)$ be stable in the sense of Dahlquist and that the roots of the polynomial $\sigma(\xi)$ with modulus equal to one be simple. Then the fully discrete problem (4.11) has one and only one solution $w_{h}$ and there exists a constant $c$ independent of $t$ and $h$ such that

$$
\begin{aligned}
& \left\|w^{s}-w_{h}^{s}\right\|_{0, \Omega \cap \Omega_{h}} \leqq c\left\{h^{k+1}\left[\sup _{(0, T)}\|w\|_{k+3, \Omega}+\sup _{(0, T)}\left\|\frac{\partial w}{\partial t}\right\|_{k+3, \Omega}\right]\right. \\
& \left.+\Delta t_{(0, T)}^{q} \sup _{\|}\left\|\frac{\partial^{q+1} w}{\partial t^{q+1}}\right\|_{k+1, \Omega}+\sum_{j=0}^{v-1}\left\|\eta_{d}^{j}-w_{h}^{j}\right\|_{h}\right\}, \quad \forall s(s \Delta t<T),
\end{aligned}
$$

where $\eta_{d}$ is the Ritz discrete approximation of the Calderon extension $\tilde{w}$ of the function $w$.

Remark 1: From (7.2) we see that the $L_{2}$-norm of the error is of a magnitude of the order $\Delta t^{q}$ with respect to $t$ and of the order $h^{k+1}$ with respect to $x$.

Remark 2: Evidently

$$
\left\|w^{m}-w_{h}^{m}\right\|_{h} \leqq\left\|w^{m}-\eta_{d}^{m}\right\|_{h}+\left\|\eta_{d}^{m}-w_{h}^{m}\right\|_{h} .
$$

R.A.I.R.O. Analyse numérique/Numerical Analysis 
Hence, from (7.27) and from the theorem on the Ritz discrete approximation [see (5.16)] it follows

$$
\begin{aligned}
\left\|w^{s}-w_{h}^{s}\right\|_{h} \leqq c\left\{h^{k+1}\left[\sup _{(0, T)}\|w\|_{k+3, \Omega}+\sup _{(0, T)}\left\|\frac{\partial w}{\partial t}\right\|_{k+3, \Omega}\right]\right. \\
\left.+\Delta t_{(0, T)}^{q} \sup \left\|\frac{\partial^{q+1} w}{\partial t^{q+1}}\right\|_{k+1, \Omega}+\sum_{j=0}^{v-1}\left\|\eta_{d}^{j}-w_{h}^{j}\right\|_{h}\right\}
\end{aligned}
$$

i.e. in (7.2) the norm $\|\cdot\|_{0, \Omega \cap \Omega_{h}}$ may be replaced by the norm $\|\cdot\|_{h}$.

Remark 3: From the Lemma 5.2 [see (5.12)] we can see that the condition (7.1) is satisfied for example in case that the quadrature formula on the reference set $\hat{K}$ for evaluation of the form $(.,)_{0, \Omega_{n}}$ is of a degree $d \geqq 2 k$. Nevertheless this condition is not necessary. Using the quadrature formula

$$
\int_{R} \varphi(\hat{x}) d \hat{x} \approx \frac{\operatorname{mes} \hat{K}}{n}[\varphi(0, \ldots, 0)+\varphi(1,0,0, \ldots, 0)+\ldots+\varphi(0, \ldots, 0,1)]
$$

(which is of degree 1) in case of 1-regular triangulation (i. e. $k=1$-linear elements) it can be proved that (7.1) is satisfied, too.

Proof of the theorem 7.1: We know that the problem (4.11) is represented by the linear system of algebraic equations (4.13). Hence, to prove the existence and the uniqueness it suffices to show that the matrix $\alpha_{v} \mathbf{M}_{h}+\Delta t \beta_{v} \mathbf{K}_{h}$ is positive definite. In the previous part we have proved that $\mathbf{K}_{h}$ is positive definite. In the case of $A_{0}$-stable methods $\alpha_{v}>0, \beta_{v}>0$. Hence it is sufficient to prove that

$$
\mathbf{m}^{T} \mathbf{M}_{h} \mathbf{m} \geqq 0
$$

for any nonzero vector $\mathbf{m}=\left[m_{1}, m_{2}, \ldots, m_{s}\right]^{T}$.

From (4.7), (5.11) and (7.1) it follows

$$
\mathbf{m}^{T} \mathbf{M}_{h} \mathbf{m}=\left(g \sum_{i=1}^{s} m_{i} \varphi_{i}, \sum_{i=1}^{s} m_{i} \varphi_{i}\right)_{h}=\left\|\sum_{i=1}^{s} m_{i} \varphi_{i}\right\|_{h}^{2} \geqq c\left\|m_{i} \varphi_{i}\right\|_{0, \Omega_{h}}^{2}>0
$$

and (7.3) is proved. More, the matrix $\mathbf{M}_{h}$ is positive definite. Let us suppose that $w \in H^{k+3}(\Omega), \forall t \in(0, T)$. By the Calderon theorem there exist extensions $\tilde{w}, \tilde{w}_{t}$ of the functions $u, \partial w / \partial t$ onto $\tilde{\Omega}$ such that

$$
\|\tilde{w}\|_{k+3, \Omega} \leqq c\|w\|_{k+3, \Omega},
$$

where $c$ is a constant independent of $h$ and $t$. Denote

$$
\hat{f}=-L \tilde{w}+G(x) \tilde{w}_{t} .
$$

vol. $13, \mathrm{n}^{\circ} 3,1979$ 
Evidently the function $\tilde{f}$ is an extension of the function $f$. Obviously

$$
\left\|\tilde{w}^{m}-w_{h}^{m}\right\|_{0 . \Omega_{h}} \leqq\left\|\tilde{w}^{m}-\eta_{d}^{m}\right\|_{0, \Omega_{n}}+\left\|\eta_{d}^{m}-w_{h}^{m}\right\|_{0, \Omega_{n}},
$$

where $\eta_{d}$ is the Ritz discrete approximation of the function $\tilde{w}$. Since $\tilde{w}(x, t)=w(x, t)=0$ on $\partial \Omega$ for every $t \in(0, T)$ we may apply the theorem on the Ritz discrete approximation. From (5.15) and (7.4) we get

$$
\left\|\tilde{w}^{m}-\eta_{d}^{m}\right\|_{0, \Omega_{n}} \leqq c h^{k+1}\left\|w^{m}\right\|_{k+3, \Omega}, \quad \forall t \in(0, T),
$$

where $c$ is a constant independent of $t$ and $h$. From (5.2) and (7.5) it follows

$$
A_{h}\left(\eta_{d}^{m}, v\right)=-\left(L \tilde{w}^{m}, v\right)_{h}=\left(\tilde{f}-G(x) \tilde{w}_{t}, v\right)_{h}, \quad \forall v \in V_{h} .
$$

Hence

$$
A_{h}\left(\eta_{d}^{m}, v\right)=\left(f^{m}, v\right)_{h}-\left(g(x) \frac{\partial w^{m}}{\partial t}, v\right)_{h}, \quad \forall v \in V_{h}
$$

Therefore from (4.11) we get for any $v \in V_{h}$

$$
\begin{gathered}
\left(g \sum_{j=0}^{v} \alpha_{j}\left(\eta_{d}^{m+j}-w_{h}^{m+j}\right), v\right)_{h}+\Delta t A_{h}\left(\sum_{j=0}^{v} \beta_{j}\left(\eta_{d}^{m+j}-w_{h}^{m+j}\right), v\right) \\
=\left(g \sum_{j=0}^{v} \alpha_{j} w^{m+j}, v\right)_{h}-\left(g \sum_{j=0}^{v} \alpha_{j}\left(w^{m+j}-\eta_{d}^{m+j}\right), v\right)_{h}-\left(g \sum_{j=0}^{v} \alpha_{j} w_{h}^{m+j}, v\right)_{h} \\
+\Delta t\left(\sum_{j=0}^{v} \beta_{j} f^{m+j}, v\right)_{h}-\Delta t\left(g \sum_{j=0}^{v} \beta_{j} \frac{\partial w^{m+j}}{\partial t}, v\right)_{h} \\
+\left(g \sum_{j=0}^{v} \alpha_{j} w_{h}^{m+j}, v\right)_{h}-\Delta t\left(\sum_{j=0}^{v} \beta_{j} f^{m+j}, v\right)_{h}
\end{gathered}
$$

Hence

$$
\begin{aligned}
\left(g \sum_{j=0}^{v} \alpha_{j}\left(\eta_{d}^{m+j}-w_{h}^{m+j}\right), v\right)_{h}+\Delta t A_{h}\left(\sum_{j=0}^{v} \beta_{j}\left(\eta_{d}^{m+j}-w_{h}^{m+j}\right), v\right) & \\
& =\left(g\left(\pi^{m}-\omega^{m}\right), v\right)_{h}, \quad \forall v \in V_{h},
\end{aligned}
$$

where

$$
\begin{gathered}
\pi^{m}=\sum_{j=0}^{v}\left(\alpha_{j} w^{m+j}-\Delta t \beta_{j} \frac{\partial w^{m+j}}{\partial t}\right) \\
\omega^{m}=\sum_{j=0}^{v} \alpha_{j}\left(w^{m+j}-\eta_{d}^{m+j}\right) .
\end{gathered}
$$


We write (7.9) in a matrix form. For this purpose, let $\mathbf{v}$ be the vector $\mathbf{v}=\mathbf{M}_{h}^{1 / 2} \varphi$, where $\varphi=\left[\varphi_{1}, \ldots, \varphi_{s}\right]^{T}$ is the vector of the basis functions. Let us set $\eta_{d}^{m}-\mathrm{w}_{h}^{m}=\left(\mathbf{e}^{m}\right)^{T} v \quad$ (notice that $\left.\eta_{d}^{m}-w_{h}^{m} \in V_{h}\right)$. Since $\left(g \boldsymbol{\varphi}, \boldsymbol{\varphi}^{T}\right)_{h}=\mathbf{M}_{h} \quad$ and $A_{h}\left(\boldsymbol{\varphi}, \boldsymbol{\varphi}^{T}\right)=\mathbf{K}_{h}$ we have $\left(g \mathbf{v}, \mathbf{v}^{T}\right)_{h}=\mathbf{I}$ and $\mathbf{A}_{h}\left(\mathbf{v}, \mathbf{v}^{T}\right)=\mathbf{M}_{h}^{-1 / 2} \mathbf{K}_{h} \mathbf{M}_{h}^{-1 / 2}$. The matrix $S_{h}=\mathbf{M}_{h}^{-1 / 2} \mathbf{K}_{h} \mathbf{M}_{h}^{-1 / 2}$ is symmetric and positive definite. Putting the components $v_{i}(i=1, \ldots, s)$ of the vector $\mathbf{v}$ for $v$ in (7.9) we get

$$
\sum_{j=0}^{v}\left(\alpha_{j} \mathbf{I}+\Delta t \beta_{j} \mathbf{S}_{h}\right) \mathbf{e}^{m+j}=\mathbf{c}_{h}^{m},
$$

where

$$
\mathbf{c}_{h}^{m}=\left(g\left(\pi^{m}-\omega^{m}\right), \mathbf{v}\right)_{h}
$$

Denote

$$
\delta_{j}(\tau)=\frac{\alpha_{j}+\beta_{j} \tau}{\alpha_{v}+\beta_{v} \tau}, \quad \mathbf{d}_{h}^{m}=\left(\alpha_{v} \mathbf{I}+\Delta t \beta_{v} \mathbf{S}_{h}\right)^{-1} \mathbf{c}_{h}^{m}
$$

(the matrix $\alpha_{v} I+\Delta t \beta_{v} S_{h}$ is positive definite). Then

$$
\sum_{j=0}^{v} \delta_{j}\left(\Delta t \mathbf{S}_{h}\right) \mathbf{e}^{m+j}=\mathbf{d}^{m}
$$

and this difference equation will be solved in the way described by Zlámal [10], pp. 355-356 who used the ideas given in Henrici [5], pp. 242-244 for ordinary differential equations. From Zlámal's result (see [10], pp. 355-356) we get

$$
\left\|\mathbf{e}^{m}\right\| \leqq c\left(\sum_{j=0}^{v-1}\left\|\mathbf{e}^{j}\right\|+\sum_{j=0}^{m-v}\left\|\mathbf{c}_{h}^{j}\right\|\right)
$$

(by $\|$.$\| we denote the Euclidean norm of a vector or of a matrix). Since$ $\left\|\eta_{d}^{m}-w_{h}^{m}\right\|_{h}^{2}=\left(g\left(\eta_{d}^{m}-w_{h}^{m}\right), \eta_{d}^{m}-w_{h}^{m}\right)_{h}=\left(g \mathbf{e}^{m^{T}} \mathbf{v}, \mathbf{v}^{T} \mathbf{e}^{m}\right)_{h}=\mathbf{e}^{m^{T}}\left(g \mathbf{v}, \mathbf{v}^{T}\right)_{h} \mathbf{e}^{m}=\left\|\mathbf{e}^{m}\right\|^{2}$ we get from (7.15):

$$
\left\|\eta_{d}^{m}-w_{h}^{m}\right\|_{h} \leqq c\left(\sum_{j=0}^{v-1}\left\|\eta_{d}^{j}-w_{h}^{j}\right\|_{h}+\sum_{j=0}^{m-v}\left\|\mathbf{c}_{h}^{j}\right\|\right) .
$$

Let $\varphi \in L_{2}(\Omega)$ be any function and let $\psi \in V_{h}$ be its orthogonal projection onto $V_{h}$ in the norm $\|\cdot\|_{h}$, i.e.:

$$
(g(\varphi-\psi), v)_{h}=0, \quad \forall v \in V_{h} .
$$

vol. $13, \mathrm{n}^{\circ} 3,1979$ 
Then

$0 \leqq\|\varphi-\psi\|_{h}^{2}=(g(\varphi-\psi), \varphi-\psi)_{h}=(g(\varphi-\psi), \varphi)_{h}=(g \varphi, \varphi)_{h}-(g \psi, \varphi)_{h}$

Hence $=(g \varphi, \varphi)_{h}-(g(\varphi-\psi), \psi)_{h}-(g \psi, \psi)_{h}=\|\varphi\|_{h}^{2}-\|\psi\|_{h}^{2}$.

$$
\|\psi\|_{h} \leqq\|\varphi\|_{h}
$$

Putting $\psi=\psi^{T} \mathbf{v}=\mathbf{v}^{T} \psi$ we get

$$
\|\psi\|_{h}^{2}=(g \psi, \psi)_{h}=\left(g \psi^{T} \mathbf{v}, \mathbf{v}^{T} \psi\right)_{h}=\psi^{T}\left(g \mathbf{v}^{T}, \mathbf{v}\right)_{h} \psi=\|\psi\|^{2}
$$

and

$$
(g \varphi, \mathbf{v})_{h}=(g \psi, \mathbf{v})_{h}=\left(g \psi, \mathbf{v}^{T}\right)_{h}^{T}=\left(\psi^{T} g \mathbf{v}, \mathbf{v}^{T}\right)_{h}^{T}=\psi
$$

Therefore from (7.19) and from (7.18) it follows

$$
\left\|(g \varphi, \mathbf{v})_{h}\right\| \leqq\|\varphi\|_{h} .
$$

Hence, from (7.13) we get

$$
\left\|\mathbf{c}_{h}^{j}\right\|=\left\|\left(g\left(\pi^{j}-\omega^{j}\right), \mathbf{v}\right)_{h}\right\| \leqq\left\|\pi^{j}-\omega^{j}\right\|_{h} .
$$

Substituting this inequality into $(7.16)$ we obtain

Evidently

$$
\left\|\eta_{d}^{m}-w_{h}^{m}\right\|_{h} \leqq c\left(\sum_{j=0}^{v-1}\left\|\eta_{d}^{j}-w_{h}^{j}\right\|_{h}+\sum_{j=0}^{m-v}\left\|\pi^{j}-\omega^{j}\right\|_{h}\right)
$$

$$
\left\|\pi^{j}-\omega^{j}\right\|_{n} \leqq\left\|\pi^{j}\right\|_{n}+\left\|\omega^{j}\right\|_{n} .
$$

To estimate $\left\|\pi^{j}\right\|_{h}$ we use the assumption that the scheme $(\rho, \sigma)$ is of order $q$. It means that for any function $y(t) \in C^{s}, s \leqq q+1$, it holds

$$
\sum_{j=0}^{v} \alpha_{j} y^{\prime}(t+j \Delta t)-\Delta t \sum_{j=0}^{v} \beta_{j} \dot{y}(t+j \Delta t)=O\left(\Delta t^{s} \max \left|y^{(s)}(t+\tau)\right|\right)
$$

Hence, from (7.10) and from the first Sobolev theorem it follows

$$
\left|\pi_{j}\right| \leqq c \Delta t^{q+1} \sup _{\Omega} \sup _{(0, T)}\left|\frac{\partial^{q+1} w}{\partial t^{q+1}}\right| \leqq c \Delta t^{q+1} \sup _{(0, T)}\left\|\frac{\partial^{q+1} w}{\partial t^{q+1}}\right\|_{k+1, \Omega}
$$

provided that the function $\partial^{q+1} w(x, t) / \partial t^{q+1}$ is continuous for every $x \in \Omega$. From (3.12) we have

$$
\left\|\pi^{j}\right\|_{h}^{2}=\left(g \pi^{j}, \pi^{j}\right)_{h}=\sum_{K \in \mathscr{\ell}_{h}} \sum_{r} \omega_{r, K} g\left(b_{r, K}\right)\left(\pi^{j}\left(b_{r, K}\right)\right)^{2} .
$$

R.A.I.R.O. Analyse numérique/Numerical Analysis 
Hence, (7.22) implies

$$
\left\|\pi^{j}\right\|_{h}^{2} \leqq c \Delta t^{2(q+1)}\left[\sup _{(0, T)}\left\|\frac{\partial^{q+1} w}{\partial t^{q+1}}\right\|_{k+1, \Omega}\right]_{K \in \mathscr{C}_{h}}^{2} \sum_{r} \omega_{r, K}
$$

From (2.5) and from (1.2) we get

$$
\begin{aligned}
\sum_{K \in \mathscr{Q}_{h}} \sum_{r} \omega_{r, K}= & \sum_{K \in \mathscr{C}_{h}} \sum_{r} \hat{\omega}_{r} J_{K}\left(\hat{b}_{r}\right) \leqq \sum_{K \in \mathscr{C}_{h}} \max _{R} J_{K}(\hat{x}) \sum_{r} \hat{\omega}_{r} \\
= & \sum_{K \in \mathscr{C}_{h}} \max _{\hat{K}} J_{K}(\hat{x}) \int_{\hat{K}} d \hat{x} \leqq \sum_{K \in \mathscr{C}_{h}} \frac{\max _{\hat{R}} J_{K}(\hat{x})}{\min _{\hat{R}} J_{K}(\hat{x})} \int_{\hat{R}} J_{K}(\hat{x}) d \hat{x} \\
& \leqq c_{0}^{2} \sum_{K \in \mathscr{C}_{h}} \int_{\hat{K}} J_{K}(\hat{x}) d \hat{x}=c_{0}^{2} \sum_{K \in \mathscr{C}_{h}} \operatorname{mes} K=c_{0}^{2} \text { mes } \Omega_{h} \leqq c_{0}^{2} \text { mes } \widetilde{\Omega} .
\end{aligned}
$$

Hence, (7.23) implies

$$
\left\|\pi^{j}\right\|_{h} \leqq c \Delta t^{q+1} \sup _{(0, T)}\left\|\frac{\partial^{q+1} w}{\partial t^{q+1}}\right\|_{k+1, \Omega} .
$$

A simple calculation gives

$$
\begin{aligned}
\omega^{m}=\sum_{j=0}^{v} \alpha_{j}\left(w^{m+j}-\eta_{d}^{m+j}\right) & \\
& =\sum_{j=1}^{v} \gamma_{j}\left[w^{m+j}-\eta_{d}^{m+j}-\left(w^{m+j-1}-\eta_{d}^{m+j-1}\right)\right]+\gamma_{0}\left(w^{m}-\eta_{d}^{m}\right),
\end{aligned}
$$

where $\gamma_{j}=\sum_{i=j}^{v} \alpha_{i}$, From the consistency of the scheme $(\rho, \sigma)$ it follows that $\gamma_{0}=0$.

Hence

$$
\begin{aligned}
&\left\|\omega^{m}\right\|_{h} \leqq c \sum_{j=1}^{v}\left\|\left(w^{m+j}-w^{m+j-1}\right)-\left(\eta_{d}^{m+j}-\eta_{d}^{m+j-1}\right)\right\|_{h} \\
& \leqq c \sum_{j=1}^{v}\left\|\left(\tilde{w}^{m+j}-\tilde{w}^{m+j-1}\right)-\left(\eta_{d}^{m+j}-\eta_{d}^{m+j-1}\right)\right\|_{h} .
\end{aligned}
$$

Evidently $\eta_{d}^{m+j}-\eta_{d}^{m+j-1}$ is the Ritz discrete approximation of the function $\tilde{w}^{m+j}-\tilde{w}^{m+j-1}$. We may apply the Theorem on the Ritz discrete approximation. From (5.16), (7.25) and from the Calderon theorem we get for $(m+j-1) \Delta t \leqq \mathscr{H}_{j}<(m+j) \Delta t$ :

$$
\begin{aligned}
\left\|\omega^{m}\right\|_{h} \leqq c h^{k+1} & \sum_{j=1}^{v}\left\|\tilde{w}^{m+j}-\tilde{w}^{m+j-1}\right\|_{k+3, \Omega} \\
& \leqq c h^{k+1} \sum_{j=1}^{v}\left\|w^{m+j}-w^{m+j-1}\right\|_{k+3, \Omega} \leqq c h^{k+1} \Delta t \sum_{j=1}^{v}\left\|\frac{\partial w^{\mathscr{H}}}{\partial t}\right\|_{k+3, \Omega} .
\end{aligned}
$$

vol. $13, \mathrm{n}^{\circ} 3,1979$ 
Hence

$$
\left\|\omega^{m}\right\|_{h} \leqq c h^{k+1} \Delta t \sup _{(0, T)}\left\|\frac{\partial w}{\partial t}\right\|_{k+3, \Omega} .
$$

From (7.21), (7.24) and (7.26) we get

$$
\begin{gathered}
\sum_{j=0}^{m-v}\left\|\pi^{j}-\omega^{j}\right\|_{h} \leqq c \sum_{j=0}^{m-v}\left[\Delta t^{q+1} \sup _{(0, T)}\left\|\frac{\partial^{q+1} w}{\partial t^{q+1}}\right\|_{h+1, \Omega}+h^{k+1} \Delta t \sup _{(0, T)}\left\|\frac{\partial w}{\partial t}\right\|_{k+3, \Omega}\right] \\
=c\left[\Delta t^{q} \sup _{(0, T)}\left\|\frac{\partial^{q+1} w}{\partial t^{q+1}}\right\|_{k+1, \Omega}+h^{k+1} \sup _{(0, T)}\left\|\frac{\partial w}{\partial t}\right\|_{k+3, \Omega}\right] \sum_{j=0}^{m-v} \Delta t \\
\leqq c T\left[\Delta t_{(0, T)}^{\sup _{(0,0}}\left\|\frac{\partial^{q+1} w}{\partial t^{q+1}}\right\|_{k+1, \Omega}+h^{k+1} \sup _{(0, T)}\left\|\frac{\partial w}{\partial t}\right\|_{k+3, \Omega}\right] .
\end{gathered}
$$

Hence, the inequality (7.20) implies

$$
\begin{aligned}
&\left\|\eta_{d}^{m}-w_{h}^{m}\right\|_{h} \leqq c\left\{\Delta t^{q} \sup _{(0, T}\left\|\frac{\partial^{q+1} w}{\partial t^{q+1}}\right\|_{k+3, \Omega}\right. \\
&\left.+h^{k+1} \sup _{(0, T)}\left\|\frac{\partial w}{\partial t}\right\|\left\|_{k+3, \Omega}+\sum_{j=0}^{v-1}\right\| \eta_{d}^{j} w_{h}^{j} \|_{h}\right\} .
\end{aligned}
$$

From (7.6), (7.7), (7.1) and from (7.27) we get (7.2).

\section{REFERENCES}

1. J. H. Bramble and S. R. Hilbert, Estimation of Linear Functionals on Sobolev Spaces with Application to Fourier Transforms and Spline Interpolation, S.I.A.M. J. Numer. Anal., Vol. 7, 1970, pp. 112-124.

2. J. H. Bramble and M. Zlámal, Triangular Elements in the Finite Element Method, Math. Comp., Vol. 24, 1970, pp. 809-820.

3. P. G. Ciarlet and P. A. Raviart, The Combined Effect of Curved Boundaries and Numerical Integration in Isoparametric Finite Element Methods, The Mathematical Foundations of the Finite Element Method with Applications to Partial Differential Equations, A. K. Azız, Ed., Academic Press, New York, 1972, pp. 409-474.

4. P. G. Ciarler, The Finite Element Method for Elliptic Problems, North-Holland, Amsterdam, 1977.

5. P. HenricI, Discrete Variable Methods in Ordinary Differential Equations, Wiley, New York-London, 1962.

6. J. NeČAs, Les méthodes directes en théorie des équations elliptiques, Masson, Paris, 1967.

7. J. Nedoma, The Finite Element solution of Parabolic Equations, Aplikace matematiky, svazek, 23, 1978, č. 6, pp. 408-438. 
8. P. A. Raviart, The Use of Numerical Integration in Finite Element Methods for Solving Parabolic Equations, Conference on Numerical Analysis, Dublin, August 14$18,1972$.

9. G. Strang, Approximation in the Finite Element Method, Numer. Math., Vol. 19, 1972, pp. 81-98.

10. M. Zlámal, Finite Element Multistep Discretizations of Parabolic Boundary Value Problems, Mathematics of Computation, Vol. 29, No. 130, April 1975, pp. 350-359.

11. M. Zlámal, Curved Elements in the Finite Element Method I. S.I.A.M. J. Numer. Anal., Vol. 10, No. 1, March 1973.

12. M. Zlámal, Curved Elements in the Finite Element Method II. S.I.A.M. J. Numer. Anal., Vol. 11, No. 2, April 1974.

13. M. Zlámal, Finite Element Methods for Parabolic Equations. Mathematics of Computation, Vol. 28, No. 126, April 1974, pp. 393-409.

14. M. Zlámal, Finite Element Methods for Nonlinear Parabolic Equations, R.A.I.R.O., Vol. 11, No. 1, 1977, pp. 93-107.

15. A. ŽENIšEK, Curved Triangular Finite $C^{m}$-Elements, Aplikace matematiky, svazek 23, 1978 , č. 5, pp. 346-377.

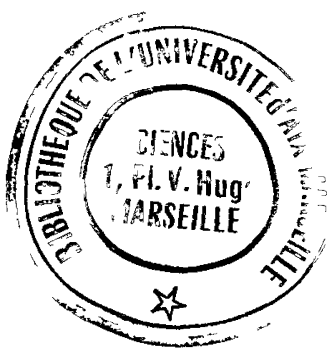

vol. $13, \mathrm{n}^{\circ} 3,1979$ 